Pacific Journal of Mathematics

THE SESSILE LIQUID DROP. I. SYMMETRIC CASE 


\section{THE SESSILE LIQUID DROP I. SYMMETRIC CASE}

\section{ROBERT FINN}

Quantitative estimates are derived, describing the size and shape of a symmetric (idealized) liquid drop, resting in gravitational equilibrium on a plane surface $\Pi$. The free surface interface is determined by the conditions that its mean curvature be a given (increasing) linear function of distance from $\Pi$, that it enclose with $I I$ a prescribed volume $V$, and that the angle formed with $\Pi$ be a prescribed constant $\gamma$. The estimates apply to drops of all sizes, and some are asymptotically exact in the limiting cases of large or small wetted area on $I$. It is shown that a number of qualitative features of behavior are determined by the ratio $V / \sin \gamma / 2$. This ratio is in turn related to a ratio that appears in the study of the circular capillary tube, thus indicating a reciprocity between the two problems, which becomes exact in both limiting cases.

As corollaries of the method, the uniqueness of the symmetric solution is proved, and a new proof of existence is given.

The results are compared with calculations and with measurements in some particular cases.

\section{Contents}

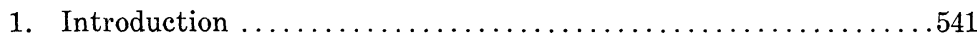

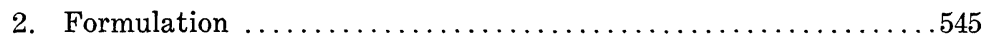

3. General Estimates ................................. 547

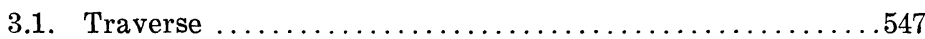

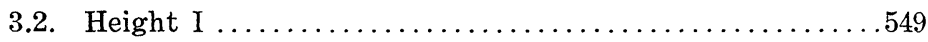

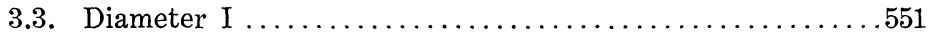

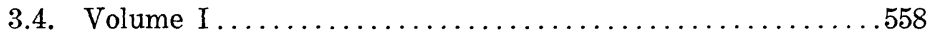

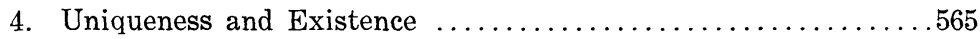

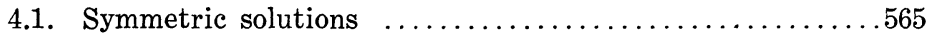

4.2. General configurations .........................567

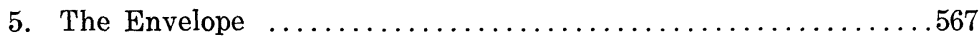

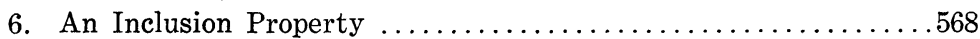

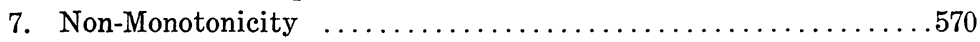

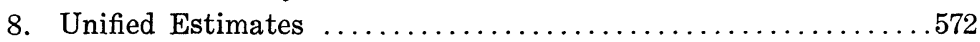

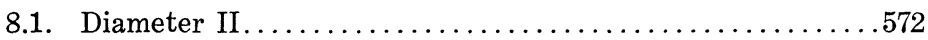

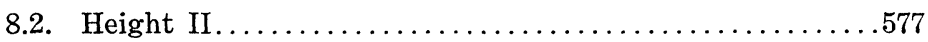

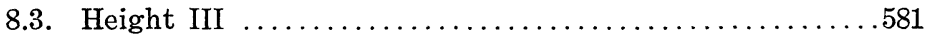

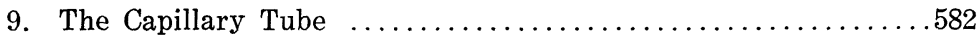

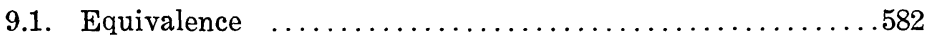

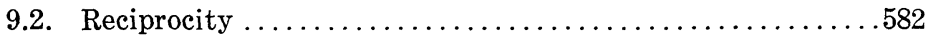

References............................................

1. Introduction. The existence of a stationary (sessile) liquid drop of prescribed volume $\mathrm{V}$ and minimizing potential energy, rest- 
ing on a homogeneous plane $\Pi$ in the presence of a uniform gravity field directed toward the plane, and making with the plane a prescribed angle $\gamma$ at the boundary of the wetted surface, has been proved by Gonzalez [6], who proved also that the drop is simply connected and rotationally symmetric relative to an axis orthogonal to the plane. (Cf. fig. 1; see also $[7,8]$.)

The result of Gonzalez leads naturally to the question, whether the solution he obtained is uniquely determined, that is, can there be more than one energy minimizing drop for prescribed $V$ and $\gamma$ ?

We show in the present work that for each $\gamma$ in the range $0<\gamma \leqq \pi$, the set of all simply connected symmetric solution surfaces is described by a one parameter family, along which $V$ varies strictly monotonically from zero to infinity. Our result, in addition to providing a new (and simpler) existence proof for equilibrium surfaces, thus settles affirmatively the above uniqueness question. In conjunction with Gonzalez's work, it shows additionally that every symmetric sessile drop is energy minimizing in the class of all drops with the same $V$ and $\gamma$.

In $\S 4.2$ we show-under some restrictions-that the symmetric configuration is unique in a class of drops that need not be symmetric or energy minimzing. ${ }^{1}$

We study here also a related question, whether, if some fluid is removed from a drop (e.g., by evaporation), the vertex height $q(V ; \gamma)$ decreases monotonically. We show in $\S 7$ that whenever the ratio $(V / \sin \gamma / 2)$ is sufficiently large, the answer is negative; it turns out the ratio $q(V ; \gamma) / \sin \gamma / 2$ tends to a constant $Q_{\infty}=$ $q_{\infty}(\gamma) / \sin \gamma / 2$, uniformly in $\gamma$ as $(V / \sin \gamma / 2) \rightarrow \infty$. Further, the ratio $q / q_{\infty}$ exceeds unity if $V / \sin \gamma / 2$ is large enough (independent of $\gamma$ ).

This latter statement turned out, to our surprise, to be not entirely new. Nonmonotonicity of $q$ in $V$ (for fixed $\gamma$ and sufficiently large $V$ ) was observed by Bowditch [10, pp. 985-6] as a consequence of a formal asymptotic expansion due to Laplace; it can be inferred also from recent work of Concus [3]. Our approach differs from those of the authors just cited, and it has the virtue, besides providing a strict mathematical proof, of showing that the controlling parameter is $(V / \sin \gamma / 2)$, rather than $V$ alone. Thus, in the case of small $\gamma$ (as occurs for water on a glass surface) monotonicity can fail for arbitrarily small values of $V$. Figure 6 shows some configurations in a neighborhood of the extremal case, ${ }^{2}$ with $\gamma=\pi / 2$ (the

${ }^{1} \mathrm{H}$. Wente has since used the same method to show that every stationary surface is necessarily symmetric.

${ }^{2}$ I am indebted to Heidi Bjorstad for performing the calculations. 
curves are sketched between the initial and end points). Relevant parameters of extremal configurations for several choices of $\gamma$ are listed in Table 1.

In $\S 6$ we show that if $(V / \sin \gamma / 2)$ is sufficiently small (we give an explicit estimate), then the height and also the "size" of the drop become monotone in $V$, in the sense that when fluid is removed symmetrically, the resulting drop lies entirely interior to the original one.

The above results-especially for the monotonicity questiondepend on general estimates for the size and shape of symmetric drops. These estimates are developed in $\S 3,8,9$. They comprise the main substance of the paper and represent, we think, its chief interest. We shall present explicit estimates, in closed form, relating the radius $r$ of the wetted surface, the volume $V$ of the drop, the boundary angle $\gamma$, the height $q$ of the drop, and the base meniscus height $u_{0}$ of a related "capillary" problem. The estimates contain some features that suggest natural lines of organization; for example, it turns out (\$8.1) that (in our normalization) the inequality $\pi / 6<$ $\left[\left(1+r^{3}\right) / r^{3}\left(1+r^{2}\right)\right] V / \sin \gamma<\pi$ holds uniformly among all possible drop configurations for which $0<\gamma \leqq \pi / 2$, and an analogous relation holds in the remaining range. Also, $\left(1 / r^{2}\right) V / \sin \gamma / 2$ is asymptotically constant for large $r$, uniformly in $\gamma$. Its deviation from a constant is estimated explicity for any $r$, and a new asymptotic relation appears as $r \rightarrow 0$. Similar comments apply to the $\operatorname{ratio}^{3}(\sin \gamma) / r u_{0}$ of the associated capillary problem; it is shown the two ratio $V / \sin \gamma$ and $(\sin \gamma) / u_{0}$ admit bounds in terms of each other for any $r$, and are determined asymptotically in terms of each other, both for small and for large $r(\S 9)$.

In general terms, we have shown that if $0<\gamma \leqq \hat{\gamma}<\pi$, then $V / \sin \gamma$ tends to zero like $\left(\sin \gamma / u_{0}\right)^{3}$, and to infinity like $\log ^{2}\left(\sin \gamma / u_{0}\right)$, as $\sin \gamma / u_{0}$ tends to the respective limits. The radius $r$ of the wetted surface (or of the capillary tube) does not enter explicitly into these asymptotic relations. Taken from another point of view, each of the ratios $V / \sin \gamma$ and $\sin \gamma / u_{0}$ determines, to within positive bounds, the radius $r$ of the wetted surface (or of an associated capillary tube), and vice versa; by adjoining multiplicative functions of $\gamma$ that are bounded above and below, the determination becomes

${ }^{3}$ The symbol $\gamma$ appearing here should be replaced by $(\pi / 2)-\gamma$ to obtain the conventional contact angle (measured within the fluid) for the capillary problem (as used in [5] and in [14]). Our choice of notation is based on the geometry of the sessile drop, for which the conventional choice for $\gamma$ has been made. This symbol will be used in the text only when a specific reference to boundary angle is intended. Generic angles measured along solution curves will be denoted by $\psi$. The notation permits each such $\psi$ to be interpreted alternatively as boundary (contact) angle. 
asymptotically exact both for small and for large values of the ratios.

Similar comments apply to the drop height $q$ above the base plane (or, equivalently, the height of the meniscus in a capillary tube). Here the crucial ratio is $q / \sin \gamma$, for which bounds above and below are obtained that become equalities asymptotically $(\S 8.2)$.

These and the previously mentioned asymptotic results seem of particular theoretical interest, both for the sessile drop and the capillary tube. The results are summarized in $\S 9$; some of the relevant quantities were also calculated independently for various choices of $r$, and appear in Table 2.

If $\gamma>\pi / 2$ (as occurs for a "nonwetting" liquid-solid interface), the methods provide estimates for the maximum diameter $R$ of the drop, and for the difference between $R$ and the radius $r$ of the wetted circle, in terms of $V$ and $\sin \gamma(\S 8.1)$.

In $\S 8.3$ we compare our estimates with the results of a formal asymptotic calculation due to Laplace, for a particular configuration that was considered by him.

The reformulation in terms of the related capillary problem has led to a number of technical simplifications, and has enabled us to profit from earliar studies of that problem in $[5,14]$. It was necessary here in the initial steps to rederive and extend for the present situation some results that were given in those references in other contexts, and thus there is a small overlap with the previously published material. The ideas are developed further here, leading to results that should be of interest also for the original capillary problem. The papers $[5,14]$ contain also a number of additional contributions to that problem, that in turn apply to the questions considered in the present work.

With the single exception of the (brief) $\S 4.2$, only the symmetric configuration is discussed here. Some of the results will however be applied to the general case (corresponding physically to an inhomogeneous base plane) in a work with J. Spruck that is now in preparation.

We have limited ourselves in this work to the case in which the total change of inclination angle of a meridional section does not exceed $\pi$, as that is the maximum change that would occur for a sessile drop. It should be pointed out there are good physical reasons to continue the study further, at least to an angle $2 \pi$, in order to include the kind of situation illustrated in figure 2. In the interest of technical simplicity we did not do so in this paper; the techniques do not all apply without change to the extended situation, however, the difficulties in basing such an 
extension on the same general frame of ideas do not seem insurmountable.

The material of this paper did not lend itself well to an organization into "Theorem" and "Proof". As a guide to the structure of the paper and to the main ideas, the numbers of principal formulas have been underlined. Italicized statements, where they appear, are used chiefly for emphasis within an immediate context. There are a few exceptions.

Figure 1 illustrates what is meant in this paper by a sessile liquid drop. A formal mathematical definition would not be difficult but for present purposes, would, we think, be needlessly pedantic. In the symmetric case we have considered, a definition is implicit in the formulation of $\S 2$. The principal interest in our results presumably lies in the (physical) case of two dimensional surfaces in three space. It requires, however, only a bit more effort to caxry out the estimates for $n$-surfaces in $(n+1)$-space, and we shall discuss the problem in that generality.

We remark finally that an independent proof of uniqueness, for the case $0<\gamma \leqq \pi / 2$, has been given by J. Spruck.

I should like to thank N. N. Ural'tseva for a conversation that attracted my attention to the problem considered here. I am indebted also to R. Gonzalez and I. Tamanini, who pointed out to me that the uniqueness question was still open.

2. Formulation. We consider a surface $S$ over a horizontal plane $\Pi$ (Figure 1) for which the energy functional is stationary. Let $p$ be a point on $S$ and $w$ a vector normal to $S$ at $p$, directed toward the exterior of the fluid region (Figure 1). If the component of $w$ orthogonal to the plane $\Pi$ is directed away from $\Pi$, we obtain, by an elementary variational analysis, the equation

$$
\operatorname{div} T u=\kappa u-\lambda, \quad T u=\frac{1}{\sqrt{1+|D u|^{2}}} D u
$$

for the distance $u(x)$ of $p$ from $\Pi$. Here $\kappa=\rho g / \sigma$ is the "capillarity constant"; $\rho$ is the density difference, $g$ the gravitational acceleration, $\sigma$ the surface tension. The constant $\lambda$ is a Lagrange parameter, to be determined by the prescribed volume. (If the component of $w$ othogonal to $\Pi$ is directed toward $\Pi$, we obtain the same equation with the signs of $\kappa$ and $\lambda$ reversed.) On the manifold of contact of $S$ with $\Pi$, the angle $\gamma$ between the normals to the surfaces is constant. The value of the constant is determined by the physical materials; we may however normalize it to the range $0<\gamma \leqq \pi$ (see Figure 1). 


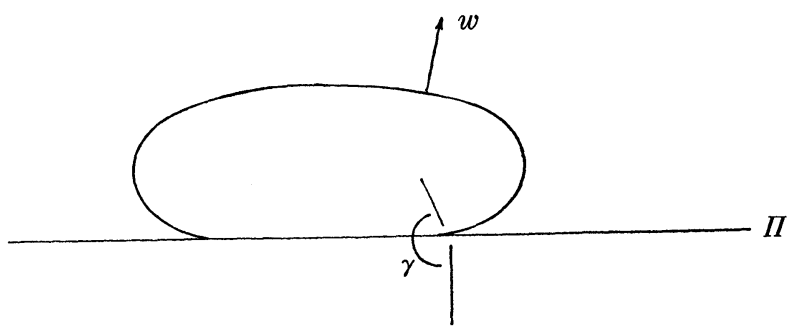

Figure 1. Sessile drop; boundary angle $\gamma$

The transformation

$$
u=\sqrt{\frac{n-1}{\kappa}} v+\frac{1}{\kappa} \lambda, \quad x=\sqrt{\frac{n-1}{\kappa}} \xi
$$

yields the equation

$$
\operatorname{div} T v=(n-1) v .
$$

We note (3) is the (normalized) equation for the rise height of fluid in a capillary tube; thus, the totality of sessile drop solutions is equivalent to the totality of capillary surfaces.

Reverting to original notation, we introduce a hypothesis of rotational symmetry about $x=0$ and set $|x|=r$, obtaining

$$
\frac{d}{d r}\left(\frac{r^{n-1} u_{r}}{\sqrt{1+u_{r}^{2}}}\right)=\frac{d}{d r}\left(r^{n-1} \sin \psi\right)=(n-1) r^{n-1} u .
$$

Here $\psi$ is the angle made with the $r$-axis by a tangent to the meridional curve $u=u(r)$ (see Figure 3). The hypotheses leading to the (necessary) condition (4) are certainly verified near the vertex of a drop, close to the point $\left(0, u_{0}\right)$ of symmetry. The existence of such a neighborhood is ensured by the following result:

For arbitrary $u_{0}$, there exists a unique solution $u(r)$ of (4) in an interval $0<r<R\left(u_{0}\right)$, and such that $\lim _{r \rightarrow 0} u(r)=u_{0}$.

This theorem (for $n=2$ ) goes back at least to Lohnstein [11]. Proofs (also for $n=2$ ) can be found also in Johnson and Perko [9], and (implicitly) in the Appendix of [4] and in [15]. The extension to arbitrary $n$ creates no difficulty. The first of the expressions in (4) reverses sign wnen the sense of the normal reverses, the second holds as stated in all cases.

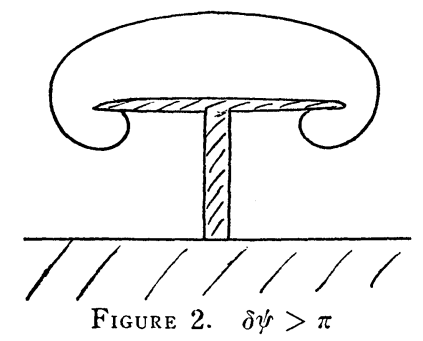




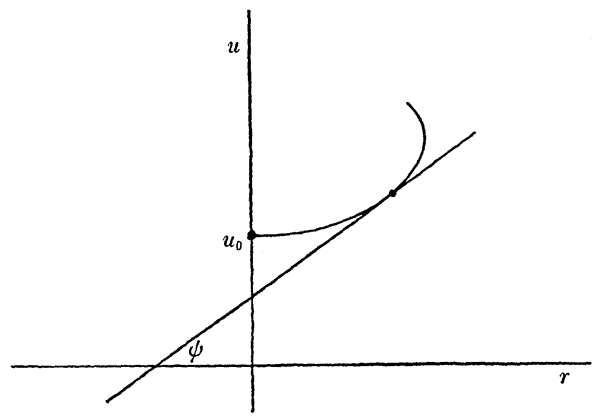

FigURE 3. Normalized configuration

We note that although (4) is of second order, it suffices (because of the singularity at $r=0$ ) to prescribe a single datum (i.e., the initial value $u_{0}$ ) to determine the solution uniquely; thus, the totality of symmetric simply connected sessile drops is determined by a one parameter family of curves. We proceed to characterize these curves qualitatively and to obtain for them various estimates. We note it suffices to consider the interval $u_{0}>0$.

\section{General estimates.}

3.1. Traverse. From (4) we find, for a given solution $u(r)$ and $0<r<R$,

$$
r^{n-1} \sin \psi=(n-1) \int_{0}^{r} \rho^{n-1} u(\rho) d \rho
$$

from which we conclude that if $u_{0}>0$, then $u(r)$ is strictly increasing in the range of $r$ for which it can be continued as a solution of (4).

Inserting upper and lower values for $u(\rho)$ into (5), we obtain the useful inequalities

$(\underline{6} a, b)$

$$
\frac{n-1}{n} u_{0}<\frac{\sin \psi}{r}<\frac{n-1}{n} u(r) .
$$

We conclude: The trajectory can be continued across the hyperbola $r u=n /(n-1)$, and develops a singularity at a value $R<n /(n-1)$ $1 / u_{0}$. The strict inequalities $(6 \mathrm{a}, \mathrm{b})$ continue to hold until $r=R$. We write (4) in the form

$$
(n-1) \frac{\sin \psi}{r}+(\sin \psi)_{r}=(n-1) u
$$

and place $(6 \mathrm{~b})$ into $(7)$ to obtain

$$
(\sin \psi)_{r}>\frac{n-1}{n} u(r)
$$


which shows that if $u_{0}>0$, then $\psi(r)$ is strictly increasing along the trajectory. We may thus introduce $\psi$ as independent variable, obtaining the parametric system

$$
\begin{aligned}
& \frac{d r}{d \psi}=\frac{r \cos \psi}{(n-1) \Delta} \\
& \frac{d u}{d \psi}=\frac{r \sin \psi}{(n-1) \Delta}
\end{aligned}
$$

with

$$
\Delta=r u-\sin \psi
$$

From (6) we find

$$
\Delta>\frac{1}{n-1} \sin \psi
$$

on $0<\psi \leqq \pi / 2$. We conclude from (9), (10), first, that $u(r)$ tends to a finite limit as $r \rightarrow R$, and then that the solution $(r, u)$ of $(\mathbf{9})$ can be continued as a solution past the value $\psi=\pi / 2$ corresponding to $r=R$.

Consider an interval $[r, R]$ into which a continuation is possible, with $\sin \psi>0$. From (4) we see there holds again on this interval

$$
\left(r^{n-1} \sin \psi\right)_{r}=(n-1) r^{n-1} u
$$

and thus, writing (imprecisely) $u(\rho)=u(\psi(\rho))$ on the continuation,

$$
R^{n-1}-r^{n-1} \sin \psi=(n-1) \int_{r}^{R} \rho^{n-1} u(\rho) d \rho .
$$

From $(9 \mathrm{~b}, \mathrm{c})$ we see that $u(\psi)$ increases monotonically on the continuation in any subinterval of $[\pi / 2, \pi]$ in which $r>0$ and (10) holds. Since these inequalities hold at $\psi=\pi / 2$, they continue to hold on some open interval including $\pi / 2$. In any such interval we find from (12) and the monotonicity of $u(\psi)$ that

$$
\begin{gathered}
\frac{n-1}{n} u(r)+\frac{R^{n-1}}{r^{n}}\left[1-\frac{n-1}{n} R u(r)\right]<\frac{\sin \psi}{r}<\frac{n-1}{n} u(r) \\
+\frac{R^{n-1}}{r^{n}}\left[1-\frac{n-1}{n} R u(R)\right] .
\end{gathered}
$$

From $(6 b)$ now follows

$$
\frac{n-1}{n} R u(R)-1>\delta>0 .
$$

We conclude at once that the solution is determined, with $r>0$, on 
the entire interval $0<\psi \leqq \pi$, that $u(\psi)$ increases monotonically and that (6b), (9), (10) hold on that interval.

On the interval $0<\psi<\pi / 2$, we may place (6b) into (9a) to obtain

$$
\frac{d r}{d \psi}<r \cot \psi
$$

from which

$$
\frac{d}{d \psi}\left(\frac{\sin \psi}{r}\right)>0, \quad 0<\psi<\pi / 2 .
$$

The same procedure on the continued solution yields

$$
\frac{d}{d \psi}\left(\frac{\sin \psi}{r}\right)<0, \quad \pi / 2<\psi<\pi .
$$

Thus, in all cases there holds

$$
\frac{\sin \psi}{r} \leqq \frac{\sin \psi_{R}}{R}=\frac{1}{R}
$$

on $0<\psi \leqq \pi$, equality holding only at $\psi=\pi / 2$.

3.2. Height I. We write $(9 \mathrm{~b})$ in the form

$$
(n-1)\left(u-\frac{\sin \psi}{r}\right) d u=\sin \psi d \psi ;
$$

we note by $(6 \mathrm{~b})$ and the monotonicity of $u(\psi)$, that $u(\psi)>n /(n-1)$ $\sin \psi / r$ in $0<\psi \leqq \pi / 2, u(\psi)>(n /(n / 1)(1 / R)$ if $\psi>\pi / 2$, and we integrate (19) in two steps, obtaining

$$
\int_{u_{0}}^{\Lambda}\left(u-\frac{\sin \tau}{\rho}\right) d u+\int_{\Lambda}^{u}\left(u-\frac{\sin \tau}{\rho}\right) d u=\frac{1-\cos \psi}{n-1}
$$

where $A$ is to be determined. To the first of these integrals we apply $(6 b)$; in the second we observe from $(17)$ that $(\sin \tau) / \rho<(\sin \psi) / r$ if $0<\tau<\psi \leqq \pi / 2$. Thus, if $0<\psi \leqq \pi / 2$, we find

$$
u(\psi)<\frac{\sin \psi}{r}+\left\{2 \frac{1-\cos \psi}{n-1}+\left(\Lambda-\frac{\sin \psi}{r}\right)^{2}-\frac{1}{n}\left(\Lambda^{2}-u_{0}^{2}\right)\right\}^{1 / 2} .
$$

The right side of (21) is minimized if $\Lambda=n /(n-1) \sin \psi / r$, a value which, by $(6 \mathrm{~b})$, lies interior to the range of integration of (20). Thus,

$$
\begin{aligned}
& u(\psi)<\frac{\sin \psi}{r}+\left\{\frac{2(1-\cos \psi)}{n-1}-\frac{1}{n-1} \frac{\sin ^{2} \psi}{r^{2}}+\frac{1}{n} u_{0}^{2}\right\}^{1 / 2}, \\
& 0<\psi \leqq \frac{\pi}{2}
\end{aligned}
$$


If we apply (6a) to (22), we obtain the estimate ${ }^{(4)}$

$$
\begin{aligned}
u(\psi)<\frac{\sin \psi}{r}+\left\{\frac{2(1-\cos \psi)}{n-1}+\frac{1}{(n-1)^{2}} \frac{\sin ^{2} \psi}{r^{2}}\right\}^{1 / 2} & , \\
0 & <\psi \leqq \frac{\pi}{2} .
\end{aligned}
$$

If $\psi>\pi / 2$, we apply (18) to $(9 \mathrm{~b})$ and integrate to obtain, with $u_{R}=u(\pi / 2)$,

$$
u(\psi)<\frac{1}{R}+\left\{\frac{-2 \cos \psi}{n-1}+\left(u_{R}-\frac{1}{R}\right)^{2}\right\}^{1 / 2}, \quad \frac{\pi}{2}<\psi \leqq \pi,
$$

which in turn implies, using - respectively - (22) or (23) at $\psi=$ $\pi / 2$,

$$
\begin{gathered}
u(\psi)<\frac{1}{R}+\left\{\frac{2(1-\cos \psi)}{(n-1)}-\frac{1}{(n-1)} \frac{1}{R^{2}}+\frac{1}{n} u_{0}^{2}\right\}^{1 / 2}, \frac{\pi}{2}<\psi \leqq \pi \\
u(\psi)<\frac{1}{R}+\left\{\frac{2(1-\cos \psi)}{n-1}+\frac{1}{(n-1)^{2}} \frac{1}{R^{2}}\right\}^{1 / 2}, \quad \frac{\pi}{2}<\psi \leqq \pi .
\end{gathered}
$$

To these relations should be adjoined

$$
u(\psi)>\left\{\begin{array}{l}
\frac{n}{n-1} \frac{\sin \psi}{r}, \quad 0<\psi \leqq \frac{\pi}{2} \\
\frac{n}{n-1} \frac{1}{R}, \frac{\pi}{2}<\psi \leqq \pi
\end{array}\right.
$$

Alternatively, we may apply $(6 \mathrm{~b})$ to the entire range of integration to obtain from $(\mathbf{9 b})$

$$
u(\psi)<\left\{\frac{2 n}{n-1}(1-\cos \psi)+u_{0}^{2}\right\}^{1 / 2}, \quad 0<\psi \leqq \pi,
$$

an estimate in which $r$ does not appear explicitly.

The same procedure using (6a) yields

$$
u(\psi)>\frac{n-1}{n} u_{0}+\left\{\frac{2}{n-1}(1-\cos \psi)+\frac{u_{0}^{2}}{n^{2}}\right\}^{1 / 2}, \quad 0<\psi \leqq \frac{\pi}{2} .
$$

Again $r$ does not appear explicitly. We may, however, obtain a stronger result, analogous to (22), by dividing the interval of integration as in (20). We then apply (6a) to the first term, and to the second term the inequality (which follows from (23))

\footnotetext{
${ }^{4}$ cf. Siegel [14].
} 
$(30)$

$$
\frac{\sin \psi}{r}>\frac{u}{1+\frac{1}{n-1} \sqrt{1+\frac{r^{2}}{k^{2}}}}, \quad 0<\psi \leqq \frac{\pi}{2}
$$

with

$$
k=\sqrt{\frac{1+\cos \psi}{2(n-1)}}
$$

and observe that if $r_{1}>r$, then the corresponding $k_{1}<k$, so that the factor involving $k$ can be replaced in the integration by its value at the upper end point. In the resulting estimate the best choice of dividing point is

$$
\Lambda=\frac{n-1+\sqrt{1+\frac{r^{2}}{k^{2}}}}{n} u_{0}
$$

which by (29) and (6a) lies interior to the interval of integration. The choice (32) leads to the estimate

$$
u(\psi)>\sqrt{\frac{p+1}{p}}\left\{\frac{2(1-\cos \psi)}{n-1}+p\left(\frac{n-1}{n} u_{0}\right)^{2}+\frac{u_{0}^{2}}{n^{2}}\right\}^{1 / 2}, 0<\psi \leqq \frac{\pi}{2},
$$

with

$$
p=\frac{1}{n-1} \sqrt{1+\frac{r^{2}}{k^{2}}} .
$$

Using (6a) in $(9 b)$ on the interval $[\psi, \pi / 2]$ yields

$$
\begin{array}{rlrl}
u(\psi)< & \frac{n-1}{n} u_{0}+\left\{\left(u_{R}-\frac{n-1}{n} u_{0}\right)^{2}-\frac{2}{n-1} \cos \psi\right\}^{1 / 2} \\
& <\left\{u_{R}^{2}-\frac{2}{n-1} \cos \psi\right\}^{1 / 2}, & 0<\psi \leqq \frac{\pi}{2},
\end{array}
$$

and using (17), we find from $(9 \mathrm{~b})$

$$
\begin{aligned}
& u(\psi)>\frac{\sin \psi}{r}+\left\{-\frac{2 \cos \psi}{n-1}+\left(u_{R}-\frac{\sin \psi}{r}\right)^{2}\right\}^{1 / 2} \\
& >\left\{u_{R}^{2}-\frac{2}{n-1} \cos \psi\right)^{1 / 2}, \\
& \frac{\pi}{2}<\psi \leqq \pi
\end{aligned}
$$

Here $u_{R}=u(\pi / 2)$ can be estimated using the preceding relations.

3.3. Diameter $I$. We need relations connecting the rdius $r$ and volume of a drop with the value $u_{0}$. An upper bound for $r$, given by (6a) when $i s \pi / 2$, is asymptotically exact for large $u_{0}$, but a 
poor estimate if $u_{0}$ is small. We obtain further information by placing (23) into (9a), yielding, for $0<\psi \leqq \pi / 2$,

$$
\frac{1}{k} \frac{\sqrt{r^{2}+k^{2}}}{r} \frac{d r}{d \psi}>\cot \psi
$$

with $k$ given by (31).

If $0<\psi \leqq \psi_{1} \leqq \pi / 2$, then

$$
\frac{1}{k_{1}} \frac{\sqrt{r^{2}+k_{1}^{2}}}{r} \frac{d r}{d \psi}>\cot \psi
$$

with $k_{1}=k\left(\psi_{1}\right)$; integrating over the interval $\left(0, \psi_{1}\right)$ and using

$$
\lim _{\psi \rightarrow 0} \frac{r(\psi)}{\sin \psi}=\frac{n}{n-1} \frac{1}{u_{0}}
$$

we are led to the inequality

$$
\frac{\rho}{1+\sqrt{1+\rho^{2}}} e^{\sqrt{1+\rho^{2}}-1}>\frac{n}{2 k(n-1)} \frac{\sin \psi}{u_{0}} ;
$$

where we have set $\rho k=r$ and removed the subscript. ${ }^{5}$

The relation (40) is a counterpart to (6a) and provides an estimate in the other direction. Taken together, (6a) and (40) show that both estimates are asymptotically exact as $r \rightarrow 0$; further, the estimate for $\sin \psi / u_{0}$ is asymptotically independent of $\psi$.

For large $r,(40)$ yields correctly an exponential growth, however the exponent is too large. We obtain a better estimate in this case by observing from (23)

$$
\frac{r u}{\sin \psi}-1<\frac{1}{n-1} \sqrt{1+\frac{2(n-1) r^{2}}{1+\cos \psi}}
$$

and also

$$
1+\frac{2(n-1) r^{2}}{1+\cos \psi}<\left[1+(n-1) r^{2}\right] \frac{2}{1+\cos \psi} ;
$$

we thus find from $(9 a)$

$$
\frac{\sqrt{1+(n-1) r^{2}}}{r} d r>\frac{\cos \psi \sqrt{1+\cos \psi}}{\sqrt{2} \sin \psi} d \psi .
$$

Integrating over the interval $(0, \psi)$ and using (39), we now find

$$
\frac{2 r}{1+\sqrt{1+\sigma^{2}}} e^{\sqrt{1+\sigma^{2}}-1}>\frac{n}{n-1} \frac{\sin \psi}{u_{0}} \times
$$

${ }^{5}$ We note that (37) leads also, in a somewhat simpler way, to an improvement in the result of 1.10 in [5]. 


$$
\times \frac{4}{1+\cos \psi+\sqrt{2(1+\cos \psi)}} e^{2(\cos \psi / 2-1)}
$$

where we have set $\sigma=\sqrt{n-1} r$. Here the exponent in the growth estimate is exact for large $r$ (cf. Siegel [14]), however, (40) is more precise for small $r$. Putting (40) and (44) together, we obtain the general result

$$
\begin{aligned}
& \frac{n}{n-1} \frac{\sin \psi}{r u_{0}}<\min \left\{\frac{2}{1+\sqrt{1+\rho^{2}}} e^{\sqrt{1+\rho^{2}}-1},\right. \\
& \left.\frac{1+\cos \psi+\sqrt{2(1+\cos \psi)}}{4} e^{2(1-\cos \psi / 2)} \frac{2}{1+\sqrt{1+\sigma^{2}}} e^{\sqrt{1+\rho^{2}}-1}\right), \\
& \rho=k^{-1} r, \quad \sigma=\sqrt{n-1 r}, \quad 0<\psi \leqq \frac{\pi}{2} .
\end{aligned}
$$

We see in particular that $\sin \psi / u_{0}$ is bounded above, depending only on the diameter $2 r$ of the wetted surface.

We note also the auxiliary inequality

(46) $\frac{1+\cos \psi+\sqrt{2(1+\cos \psi)}}{4} e^{2(1-\cos \psi / 2)} \leqq \frac{1+\sqrt{2}}{4} e^{2-\sqrt{2}}=1.084$

which yields a convenient simplified form of (45).

We consider the interval $\pi / 2<\psi \leqq \pi$. From (18) we have immediately

$$
r>R \sin \psi
$$

with a lower bound for $R$ available from (27) or from (40): Alternatively, we find from (9a), if $\psi>\pi / 2$,

$$
(n-1) \frac{d r}{d \psi}>\frac{r \cos \psi}{r u_{R}-\sin \psi}
$$

which can be rewritten in the form

$$
\frac{d}{d \psi}\left[\frac{n-1}{n} r^{n} u_{R}-r^{n-1} \sin \psi\right]>0
$$

so that

$$
r^{n}-\frac{n}{n-1} \frac{\sin \psi}{u_{R}} r^{n-1}>R^{n}-\frac{n}{n-1} \frac{1}{u_{R}} R^{n-1}
$$

and $(6 \mathrm{~b})$ now implies

$$
r>\frac{n}{n-1} \frac{\sin \psi}{u_{R}}, \quad \frac{\pi}{2}<\psi<\pi
$$


which is in turn an improvement of (6b). In (51), $u_{R}$ can be estimated using (28). (The estimates obtained from (22) or (23) are already contained in the simpler form (18).)

If we insert the inequality $r^{n-1}>r^{n} R^{-1}$ into (50), we are led to the additional estimate, for $\pi / 2 \leqq \psi \leqq \pi$,

$$
r^{n}>R^{n} \frac{\frac{n-1}{n} R u_{R}-1}{\frac{n-1}{n} R u_{R}-\sin \psi}
$$

which improves on (18) and (51) for values of $\psi$ near $\pi$.

We note from $(29)$ there holds always $u_{R}>(2 / n-1)^{1 / 2}$, thus

$$
r^{n}>R^{n} \frac{R \sqrt{2(n-1)}-n}{R \sqrt{2(n-1)}-n \sin \psi}
$$

which yields a nontrivial estimate whenever $R \sqrt{2(n-1)}>n$. Letting $r_{T}=r(\pi)$ be the first positive $r$ at which the surface becomes horizontal, we obtain

$$
r_{T}>R\left(1-\frac{n}{\sqrt{2(n-1)}} \frac{1}{R}\right)^{1 / n}
$$

and thus

$$
r_{T}>R-\frac{1}{\sqrt{2(n-1)}}
$$

for all sufficiently large $R$. The estimates $(54,55)$ provide, to our knowledge, the first explicit information on the diameter of the wetted surface for a symmetric sessile drop. They are not the best estimates obtainable by the method; they could be made more precise, for example, by using the full strength of (29) in (52), or of (36) in the derivation of $(50)$. It will turn out $(\S 8)$ that $R$ can be estimated in terms of volume.

We turn now to the question of bounding $r(\psi)$ from above; as before we study first the interval $0<\psi \leqq \pi / 2$, and we note (6a) yields the estimate

$$
r<\frac{n}{n-1} \frac{\sin \psi}{u_{0}}
$$

which is asymptotically exact as $\sin \psi / u_{0} \rightarrow 0$.

We obtain a better result by introducing (33) into (9a). To do so, we note first that 


$$
\sqrt{1+\frac{1}{p}}>1+\frac{1}{2 p}-\frac{1}{8 p^{2}}
$$

We have

$$
\frac{1}{n-1} \frac{r}{k}<p=\frac{1}{n-1} \sqrt{1+\frac{r^{2}}{k^{2}}}<\frac{1}{n-1} \frac{r}{k}\left(1+\frac{k^{2}}{2 r^{2}}\right)
$$

and thus

$$
\sqrt{1+\frac{1}{p}}>1+\frac{n-1}{2} \frac{k}{r}-\frac{(n-1)^{2}}{2} \frac{k^{2}}{r^{2}}
$$

whenever the inequality

$$
2 r^{2}>k^{2}
$$

holds.

We have from (33)

$$
r u(\psi)>\frac{1}{n-1} \sqrt{1+\frac{1}{p}} \frac{r}{k} \sin \psi
$$

and hence, using (57) and noting $(1+\cos \psi)<2$,

$$
\begin{aligned}
\frac{n-1}{r}\left(\frac{r u}{\sin \psi}\right. & -1)>\sqrt{\frac{2}{1+\cos \psi}} \\
& \times\left(\sqrt{n-1}-\frac{n-1}{2 r}-\frac{(n-1)^{3 / 2}}{2} \frac{1}{r^{2}}\right)
\end{aligned}
$$

whenever (58) holds. We place this estimate into (9a) and integrate between limits $s$ and $r$ to obtain

$$
\begin{aligned}
& \sqrt{n-1}(r-s)-\frac{n-1}{2} \log \frac{r}{s} \\
& \quad+\frac{(n-1)^{3 / 2}}{2}\left(\frac{1}{r}-\frac{1}{s}\right)<\left[2 \cos \frac{\psi}{2}+\log \tan \frac{\psi}{4}\right] \psi_{\psi_{s}}^{\psi_{r}} .
\end{aligned}
$$

Choosing

$$
s=\frac{1}{2} \frac{n+1}{\sqrt{n-1}},
$$

we note that (58) holds for this choice, and using (6a) we are led after some manipulation to

$$
\frac{n}{n-1} \frac{\sin \psi}{u_{0}}>A(n ; \psi ; r ; s) r^{-(n-1) / 2} e^{\sqrt{n-1} r}, \quad r>s ; 0<\psi \leqq \pi / 2,
$$


with

$$
\begin{aligned}
& A(n ; \psi ; r ; s)=\left(\frac{1}{2} \frac{n+1}{\sqrt{n-1}}\right)^{(n+1) / 2} \frac{e^{2 \cos \left(\psi_{s} / 2\right)}}{\left.1+\cos \psi_{s}+\sqrt{2\left(1+\cos \psi_{s}\right.}\right)} \\
& \quad \times \exp \left\{-\frac{(n-1)^{2}}{n+1}-\frac{n+1}{2}\right\} \frac{1+\cos \psi+\sqrt{2(1-\cos \psi)}}{e^{2 \cos (\psi / 2)}} \\
& \quad \times \exp \left\{\frac{(n-1)^{3 / 2}}{2 r}\right\} .
\end{aligned}
$$

There holds uniformly, for all $r>s$,

(65) $A(n ; \psi ; r ; s)>A_{0}=\left(\frac{1}{2} \frac{n+1}{\sqrt{n-1}}\right)^{n+1 / 2} \exp \left\{-\frac{(n-1)^{2}}{n+1}-\frac{n+1}{2}\right\}$.

In any range $r \geqq 2 s$, this estimate can be improved. We may observe first that the left side of (61) is increasing in $r$, and thus, for $r \geqq 2 s$,

$$
\begin{gathered}
\sin \psi_{s}<2^{(n-1) / 2} e^{-2 n /(n+1)} \frac{\cos ^{2}\left(\psi_{s} / 2\right)+\cos \left(\psi_{s} / 2\right)}{e^{2 \cos \left(\psi_{s} / 2\right)}} \\
\times \frac{e^{2 \cos (\psi / 2)}}{\cos ^{2}(\psi / 2)+\cos (\psi / 2)} \sin \psi
\end{gathered}
$$

from which

$$
\sin \psi_{s}<2^{n-1 / 2} e^{-2 n / n+1} \sin \psi=\lambda_{n} \sin \psi
$$

and hence

$$
\cos \frac{\psi_{s}}{2}>\frac{1}{\sqrt{2}}\left[1+\sqrt{1-\lambda_{n}^{2} \sin ^{2} \psi_{s}}\right]^{1 / 2}
$$

This estimate can now be put into (65.1) to improve the bound on $\sin \psi_{s}$, and so forth. The sequence of values obtained is monotone, hence convergent.

We illustrate the procedure in the case $n=2$, for which $\lambda_{2}=$ $\sqrt{2} e^{-4 / 3}=.373$. Thus, by (65.2), $\sin \psi_{s}<.373 \sin \psi \leqq .373$ so that, by (65.3), $\cos \left(\psi_{s} / 2\right)>0.982$. From (65.1) we now find

$$
\begin{aligned}
\sin \psi_{s} & <\sqrt{2} e^{-4 / 3} \times .546 \times \frac{e^{2 \cos (\psi / 2)}}{\cos ^{2}(\psi / 2)+\cos (\psi / 2)} \sin \psi \\
& <\sqrt{2} e^{-4 / 3} \times .546 \times \frac{2 e^{\sqrt{2}}}{1+\sqrt{2}} \\
& =0.347
\end{aligned}
$$

from which, by (65.3), 


$$
\cos \frac{1}{2} \psi_{s}>0.984
$$

and so forth. Thus, if $r \geqq 2 s$, there holds always

$$
\begin{aligned}
A(2 ; \psi ; r ; s) & >1.077 \frac{\cos ^{2}(\psi / 2)+\cos (\psi / 2)}{e^{2 \cos (\psi / 2)}} \exp \left\{\frac{1}{2 r}\right\} \\
& >0.294 \exp \left\{\frac{1}{2 r}\right\},
\end{aligned}
$$

the latter estimate being the one given by (65). For given $\psi$, the estimate can be further improved by using the value for $\sin \psi$ (instead of the approximation $\sin \psi<1$ ) in the above derivation.

The relation (63) complements (6a) for values $r>s$, and shows that for solutions with large wetted area, $u_{0} / \sin \psi$ becomes exponentially small in $r$.

The above result, considered as an upper bound for $u_{0}$, can be inserted into (22), and the resulting inequality placed into (9a) to obtain an improved version of (45); similarly, other of our inequalities can be sharpened by that type of iteration. The results obtained in that way are however of marginal interest for the purposes of this paper, and we do not pursue them further here.

To study the case $\psi>\pi / 2$, we observe that for any $\hat{\psi}$ with $\pi / 2 \leqq \hat{\psi} \leqq \pi$ there holds by $(26)^{6}$

$$
u(\psi)<\frac{1}{R}+\left\{\frac{2(1-\cos \hat{\psi})}{n-1}+\frac{1}{(n-1)^{2}} \frac{1}{R^{2}}\right\}^{1 / 2}=\lambda_{R}(\hat{\psi})
$$

for all $\psi$ in $\pi / 2 \leqq \psi \leqq \hat{\psi}$. Thus by $(9 \mathrm{a})$

$$
(n-1) \frac{d r}{d \psi}<\frac{r \cos \psi}{r \lambda_{R}(\hat{\psi})-\sin \psi}, \quad \frac{\pi}{2} \leqq \psi \leqq \hat{\psi},
$$

from which

$$
\frac{d}{d \psi}\left[r^{n-1} \sin \psi-\frac{n-1}{n} r^{n} \lambda_{R}\right]>0, \quad \frac{\pi}{2} \leqq \psi \leqq \hat{\psi},
$$

so that, since $\hat{\psi}$ is arbitrary in its interval,

$$
r^{n}-\frac{n}{n-1} \frac{\sin \psi}{\lambda_{R}(\psi)} r^{n-1}<R^{n}-\frac{n}{n-1} \frac{1}{\lambda_{R}(\psi)} R^{n-1}
$$

in $\pi / 2 \leqq \psi \leqq \pi$.

We now write (52) in the form

${ }^{6}$ We could improve the following estimates somewhat by using (25) instead of (26) and by estimating $u_{0}$ by (58) or by (63). 


$$
r(\psi)>R\left(\frac{\frac{n-1}{n} R u_{R}-1}{\frac{n-1}{n} R u_{R}-\sin \psi}\right)^{1 / n}=R-\eta(R ; \psi)
$$

and place the inequality

$$
r^{n-1}=\frac{1}{r} r^{n}<\frac{r^{n}}{R-\eta(R ; \psi)}
$$

into (69) to obtain

$$
r^{n}<R^{n} \frac{1-\frac{n}{n-1} \frac{1}{R \lambda_{R}(\psi)}}{1-\frac{n}{n-1} \frac{\sin \psi}{(R-\eta) \lambda_{R}(\psi)}} .
$$

Again the estimate for $r_{T}$ is of special interest. We find

$$
r_{T}<R\left(1-\frac{n}{n-1} \frac{1}{R \lambda_{R}(\psi)}\right)^{1 / n}
$$

and thus, for all sufficiently large $R$,

$$
r_{T}<R-\frac{1}{2 \sqrt{n-1}}
$$

This estimate should be compared with (55).

3.4. Volume $I$. We wish to estimate the volume $V(\psi)$ enclosed by a drop, this is, the volume cut off by a horizontal plane that meets the surface in an angle $\psi$. We have immediately by (4)

$$
\begin{aligned}
V(\psi) & =\sigma_{n} r^{n} u-\partial \sigma_{n} \int_{0}^{\psi} \rho^{n-1} u \frac{d \rho}{d \psi} d \psi \\
& =\sigma_{n} r^{n} u-\frac{\partial \sigma_{n}}{n-1} \int_{0}^{\psi} \frac{d}{d \psi}\left(\rho^{n-1} \sin \psi\right) d \psi
\end{aligned}
$$

or

$$
V(\psi)=\sigma_{n}\left(r^{n} u-\frac{n}{n-1} r^{n-1} \sin \psi\right), \quad 0 \leqq \psi \leqq \pi,
$$

where $\sigma_{n}, \partial \sigma_{n}$ are respectively the volume and surface of a unit $n$-ball. ${ }^{7}$

From the inequality (23) we obtain, for $0<\psi \leqq \pi / 2$,

$$
V(\psi)<\frac{1}{n-1} \sigma_{n} r^{n-1}\left\{\left(1+\frac{r^{2}}{k^{2}}\right)^{1 / 2}-1\right\} \sin \psi
$$

${ }^{7}$ We note that the difference between the two sides of (6b) appears as a factor in the expression for $V$. 
and thus, since $(1+x)^{1 / 2}<1+x^{1 / 2}$ if $x>0$,

$$
V(\psi)<\frac{1}{n-1} \frac{\sigma_{n}}{k} r^{n} \sin \psi, \quad 0<\psi \leqq \pi / 2,
$$

a simple estimate that provides useful information when $r$ is large.

If $r>s$ (cf. (62)), we may apply (63), (65) in (22) to obtain the sharper result

$$
\begin{array}{r}
V(\psi)<\frac{n}{n-1} \sigma_{n} r^{n-1} \sin \psi\left\{\left[\frac{r^{2}}{k^{2}}-(n-1)+\frac{1}{A_{0}} r^{n-1} e^{-2 \sqrt{n-1} r}\right]^{1 / 2}-1\right\}, \\
0<\psi \leqq \frac{\pi}{2},
\end{array}
$$

which now implies

$$
\begin{aligned}
V(\psi)< & \frac{1}{n-1} \frac{\sigma_{n}}{k} r^{n} \sin \psi\left(1-\frac{k}{r}-\frac{n-1}{2} \frac{k^{2}}{r^{2}}\right. & \\
& \left.+\frac{1}{2 A_{0}} k^{2} r^{n-3} e^{-2 \sqrt{n-1} r}\right), & 0<\psi \leqq \frac{\pi}{2} .
\end{aligned}
$$

Analogous estimates can be obtained for the case $\psi>\pi / 2$ by using (24), (25), (26) or (28); they take however a somewhat more complicated form.

We note that although the volume estimated by (75-78) is an $(n+1)$-dimensional quantity, $r$ appears in these relations only to the $n$th power. This reflects the tendency of the drop to flatten over most of its diameter and to approach a limiting height with increasing $r$.

If $r$ is small, we may use the inequality $(1+x)^{1 / 2}<1+x / 2$ in (75) to obtain

$$
V(\psi)<\sigma_{n} r^{n+1} \frac{\sin \psi}{1+\cos \psi}, \quad 0<\psi \leqq \frac{\pi}{2} .
$$

We can in some respects do better, and remove the restriction on $\psi$. Consider a sphere $\Sigma$ defined by $v\left(\psi ; u_{0}\right)$, centered on the axis of symmetry at the height $u^{\Sigma}=u_{0}+n /(n-1) 1 / u_{0}$, and of radius $r^{\Sigma}=n /(n-1) 1 / u_{0}$.

LEMma. The entire traverse of the solution $u\left(\psi ; u_{0}\right)$, in the range $0<\psi \leqq \pi$, lies interior to $\Sigma$.

Proof. In the interval $0<\psi \leqq \pi / 2$, since $\Sigma$ has constant mean curvature $[(n-1) / n] u_{0}$, the function $v\left(\varphi ; u_{0}\right)$ is determined by the equations 


$$
\begin{aligned}
\left(r^{n-1} \sin \varphi\right)_{r} & =(n-1) r^{n-1} u_{0} \\
\sin \varphi & =\frac{v_{r}}{\sqrt{1+v_{r}^{2}}} .
\end{aligned}
$$

For the solution $u\left(\psi ; u_{0}\right)$ we have

$$
\left(r^{n-1} \sin \psi\right)_{r}=(n-1) r^{n-1} u
$$

and thus

$$
r^{n-1}(\sin \psi-\sin \varphi)=(\mathrm{n}-1) \int_{0}^{r} \rho^{n-1}\left(u-u_{0}\right) d \rho .
$$

Since $u>u_{0}$, we have $\sin \psi>\sin \varphi$, hence $u^{\prime}(r)>v^{\prime}(r)$, and since both curves coincide initially at the height $u_{0}$, there follows $u>v$ in the interval $0<r \leqq R$.

We next observe that for all $\psi$ in $0<\psi \leqq \pi$, there holds $u\left(\psi ; u_{0}\right)<v\left(\psi ; u_{0}\right)$. In fact,

$$
v\left(\psi ; u_{0}\right)=u_{0}+\frac{n}{n-1} \frac{1-\cos \psi}{u_{0}},
$$

while by (28),

$$
u\left(\psi ; u_{0}\right)<\left\{\frac{2 n}{n-1}(1-\cos \psi)+u_{0}^{2}\right\}^{1 / 2},
$$

from which the stated inequality follows.

From the above two remarks we conclude at first that all points up to the first vertical of $u\left(\psi ; u_{0}\right)$ lie in $\Sigma$. Since $r\left(\psi ; u_{0}\right)$ decreases

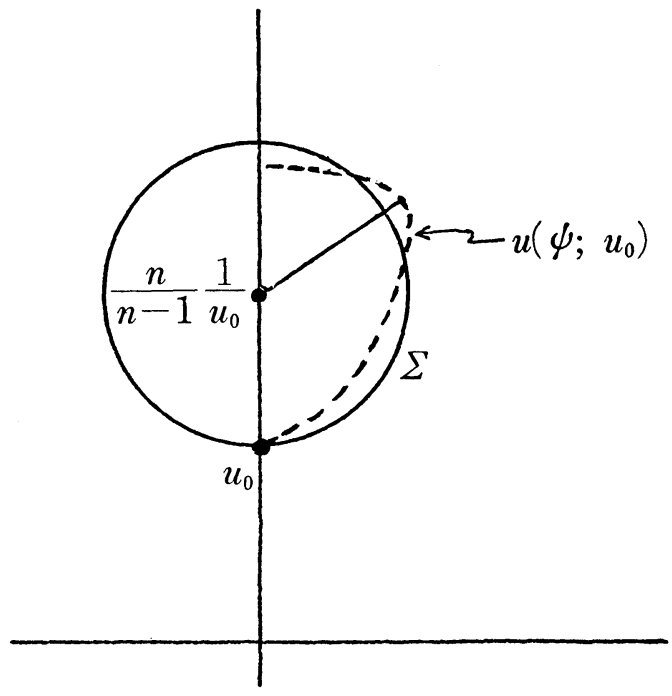

Figure 4. Proof of lemma 
for $\psi>\pi / 2$, the traverse continues to lie in $\Sigma$ until the value $\hat{\psi}$ at which $u\left(\hat{\psi} ; u_{0}\right)=u_{0}+n /(n-1) 1 / u_{0}$, i.e., at which the traverse crosses the horizontal diameter of $\Sigma$. (If there is no such $\hat{\psi}$ in $0<\psi \leqq \pi$, then there is nothing further to prove.)

Suppose there were a point $\left(r\left(\psi ; u_{0}\right), u\left(\psi ; u_{0}\right)\right), \hat{\psi}<\psi \leqq \pi$, not in $\Sigma$. Then there would be a value $\psi^{*}$ in this interval, at which the radial distance from the center of $\Sigma$ would be a maximum (see Fig. 4). There cannot hold $\psi^{*}=\pi$, since the solution curve has zero slope at this point and therefore there would be neighboring points of larger radial distance. Hence $\hat{\psi}<\psi^{*}<\pi$, and we conclude, since the solution curve lies on or outside $\Sigma$ at this point, that $u\left(\psi^{*} ; u_{0}\right) \geqq v\left(\psi^{*}: u_{0}\right)$. We have however shown above the reverse inequality; this completes the proof of the lemma.

From the lemma we conclude that the volume $V$ determined by $u\left(\psi ; u_{0}\right)$ is less than the volume $V^{\Sigma}$ of the sector $\Sigma$ up to the height $u$. Thus

$$
V(\psi)<V^{\Sigma}=\sigma_{n}\left(\frac{n}{n-1} \frac{1}{u_{0}}\right)^{n+1} \int_{0}^{\hat{\theta}} \sin ^{(n+1)} \theta d \theta
$$

with

$$
\hat{\theta}=\cos ^{-1}\left(1-\frac{n-1}{n} u_{0}\left(u-u_{0}\right)\right) .
$$

According to the above remarks, there holds $\hat{\theta}<\psi$, and thus

$$
\begin{aligned}
\frac{V(\psi)}{\sin \psi}<\sigma_{n}\left(\frac{n}{n-1} \frac{\sin \psi}{u_{0}}\right)^{n+1} \frac{1}{\sin ^{(n+2)} \psi} \int_{0}^{\psi} \sin ^{(n+1)} \theta d \theta, & \\
& 0<\psi \leqq \pi .
\end{aligned}
$$

A convenient estimate for the integral is obtained by setting

$$
\begin{gathered}
f_{n}(\psi)=\frac{1}{\sin ^{n} \psi} \int_{0}^{\psi} \sin ^{n} \theta d \theta \\
g_{n}(\psi)=\int_{0}^{\psi} \sin ^{n} \theta d \theta-\frac{\sin ^{(n+1)} \psi}{n+1} .
\end{gathered}
$$

Then $f(0)=g(0)=0$, and

$$
\begin{gathered}
f_{n}^{\prime}(\psi)=1-\frac{n \cos \psi}{\sin ^{(n+1)} \psi} \int_{0}^{\psi} \sin ^{n} \theta d \theta \\
g_{n}^{\prime}(\psi)=\sin ^{n} \psi(1-\cos \psi)>0
\end{gathered}
$$

and hence $g_{n}(\psi)>0$ and, for $0<\psi \leqq \pi / 2$, 


$$
\begin{aligned}
& f_{n}^{\prime}(\psi)<1-\frac{n}{n+1} \cos \psi \\
& f_{n}(\psi)<\psi-\frac{n}{n+1} \sin \psi
\end{aligned}
$$

Thus, (75) implies

$$
V<\sigma_{n}\left(\frac{n}{n-1} \frac{\sin \psi}{u_{0}}\right)^{n+1}\left(\psi-\frac{n+1}{n+2} \sin \psi\right), \quad 0<\psi \leqq \frac{\pi}{2},
$$

an estimate suitable chiefly for small values of $\psi$.

If $n$ is even (which includes the physical case $n=2$ ), one has the identity ${ }^{8}$

$$
\begin{aligned}
& \frac{1}{\sin ^{(n+2)} \psi} \int_{0}^{\psi} \sin ^{(n+1)} \theta d \theta \\
& \quad=\frac{1}{(1+\cos \psi)^{1+\nu}} \sum_{j=0}^{\nu}\left(\begin{array}{l}
\nu \\
j
\end{array}\right) \frac{2^{\nu-j}(1-\cos \psi)^{j}}{(1+\nu+1+j)}=A_{n}(\psi),
\end{aligned}
$$

with $\nu=n / 2$. Thus, for even $n$, (85) can be written

$$
\frac{V(\psi)}{\sin \psi}<\sigma_{n}\left(\frac{n}{n-1} \frac{\sin \psi}{u_{0}}\right)^{n+1} A_{n}(\psi)
$$

If $\psi$ is bounded from zero, a convenient expression is

$$
\begin{array}{r}
V<\sigma_{n}\left(\frac{n}{n-1} \frac{\sin \psi}{u_{0}}\right)^{n+1}\left[\frac{\sqrt{\pi}}{2} \frac{\Gamma\left(\frac{n}{2}+1\right)}{\Gamma\left(\frac{n+3}{2}\right)}+\int_{\pi / 2}^{\psi} \sin ^{(n+1)} \theta d \theta\right] \\
0<\psi \leqq \pi
\end{array}
$$

For vanishingly small values of $r$ (or, equivalently, of $\sin \psi / u_{0}$ ), we may use (40) in (86) to obtain an inequality of the form

$$
V<\sigma_{n}\left(\frac{2 r}{2-\varepsilon}\right)^{n+1}\left(\psi-\frac{n+1}{n+2} \sin \psi\right), \quad 0<\psi \leqq \pi / 2
$$

thus permitting a direct comparison with (79) in this case.

To bound $V(\psi)$ below, we consider first the case $\sin \psi / u_{0} \gg 0$; we insert (33) into (74) and use (63), (65) to obtain, for $0<\psi \leqq \pi / 2$,

$$
\begin{aligned}
V(\psi) & >\frac{1}{n-1} \frac{\sigma_{n}}{k} r^{n} \sin \psi \\
\times & \left\{\sqrt{\frac{p+1}{p}}\left[1+\frac{1+p(n-1)^{2}}{A_{0}^{2}} r^{n-1} e^{-2 \sqrt{n-1} r}\right]^{1 / 2}-\frac{k n}{r}\right\}
\end{aligned}
$$

${ }^{8} \mathrm{I}$ am indebted to $\mathrm{H}$. Wente for a simple proof. 
which should be compared with (78). Analogous estimates follow for $\psi>\pi / 2$ by adjoining (36) to (33). We note that for large $r$, (78) and (91) bound the ratio $V / \sin \psi$ above and below, depending only on the radius $r$ of the wetted disk; equivalently, they bound $r$ above and below depending only on $V / \sin \psi$, whenever that ratio is large. Both estimates can be expressed in terms of $\left(\sin \psi / u_{0}\right)$ by using, e.g., (45) or (63).

If $\sin \psi / u_{0}$ is small, we proceed somewhat differently, and compare with the volume subtended by a suitably inscribed spherical surface. We suppose first $0<\psi \leqq \pi / 2$, and we note that the meridional curve $v(r)$ of a lower hemisphere $\Sigma$, centered on the $u$-axis at the height $v_{\psi}=u_{0}+n /(n-1) 1 / u_{\psi}$ (with $u_{\psi}=u(\psi)$ ) and having radius

$$
r_{\Sigma}=\frac{n}{n-1} \frac{1}{u_{\psi}}
$$

satisfies the equation

$$
\left(r^{n-1} \sin \varphi\right)_{r}=(n-1) r^{n-1} u_{\psi}
$$

where $\varphi$ is the angle subtended at the $r$-axis by the line tangent to the curve, and thus

$$
r^{n-1}(\sin \varphi-\sin \psi)=\int_{0}^{r} \rho^{n-1}\left(u_{\psi}-u\right) d \rho .
$$

Since the inital points coidcide at $u_{0}$, it follows that $\Sigma$ lies entirely above the solution surface, at least until the height $u_{\psi}$. Let $V^{\Sigma}$ be the volume of $\Sigma$ if $\Sigma$ lies below the plane $u=u_{\psi}$, otherwise denote by $V^{\Sigma}$ the volume of that part of $\Sigma$ for which $v \leqq u_{\psi}$. We then have $V>V^{\Sigma}$, and hence, in the former case,

$$
V>V^{\Sigma}=\frac{1}{2} \sigma_{n+1} r_{\Sigma}^{n+1}=\sigma_{n} r_{\Sigma}^{n+1} \int_{0}^{\pi / 2} \sin ^{n+1} \theta d \theta .
$$

In the latter case, we have $r_{\Sigma}>u_{\psi}-u_{0}$; we find

$$
V^{\Sigma}=\sigma_{n} r_{\Sigma}^{n+1} \int_{0}^{\theta \psi} \sin ^{n+1} \theta d \theta
$$

with

$$
\theta_{\psi}=\cos ^{-1}\left(1-\frac{u_{\psi}-u_{0}}{r_{\Sigma}}\right) .
$$

To estimate this latter term, we note that if we set 


$$
P_{n}=\left\{1+\frac{2 n^{2}(1-\cos \psi)}{(n-1) u_{0}^{2}}\right\}^{1 / 2},
$$

then (29) implies

$$
\frac{u_{\psi}-u_{0}}{r_{\Sigma}}>\frac{1}{1+p_{n}} \frac{2(1-\cos \psi)}{u_{0}} u_{\psi}
$$

which yields, applying (29) again,

$$
\frac{u_{\psi}-u_{0}}{r_{\Sigma}}>\frac{2(1-\cos \psi)}{n} \frac{(n-1)+p_{n}}{1+p_{n}}
$$

and hence

$$
\frac{u_{\psi}-u_{0}}{r_{\Sigma}}>\frac{2}{n}(1-\cos \psi)
$$

so that, by (97)

$$
\cos \theta_{\psi}<1-\frac{2}{n}(1-\cos \psi)
$$

If $n=2$, we find $\theta_{\psi}>\psi$ (which means $\Sigma$ cannot lie below the plane $u=u_{\psi}$ ) and hence

$$
V>V^{\Sigma}>\sigma_{n} r_{\Sigma}^{3} \int_{0}^{\psi} \sin ^{3} \theta d \theta
$$

In all cases, we find

$$
V>V^{\Sigma}>a_{n}(\psi) r_{\Sigma}^{n+1}=a_{n}(\psi)\left(\frac{n}{n-1}\right)^{n+1} \frac{1}{u_{\psi}^{n+1}}
$$

with

$$
a_{n}(\psi)=\sigma_{n} \int_{0}^{\hat{\psi}} \sin ^{n+1} \theta d \theta
$$

and

$$
\hat{\psi}=\cos ^{-1}\left[1-\frac{2}{n}(1-\cos \psi)\right] .
$$

Here $u_{\psi}$ can be estimated from e.g., (22), (23), or (28).

We may note the general inequality

$$
\cos x>1-\frac{x^{2}}{2}
$$

implies 


$$
\hat{\psi}>\left\{\frac{4}{n}(1-\cos \psi)\right\}^{1 / 2}
$$

in all cases.

We can strengthen this result in the limiting situation of small $V / \sin \psi$. We note (104) can be expressed as an inequality of the form

$$
\frac{\sin \psi}{u_{0}}<f\left(\frac{V}{\sigma \sin \psi} ; k\right)
$$

with $\lim _{t \rightarrow 0} f(t ; k)=0$, uniformly in $k$. (The function $f$ is easily given explicitly). It follows then by a double application of (29) that for any $\varepsilon>0$,

$$
\frac{u_{\psi}-u_{0}}{r_{\Sigma}}>(1-\varepsilon)(1-\cos \psi)
$$

whenever $V / \sin \psi$ is small enough.

The relation (106) is now replaced by

$$
\hat{\psi}=\cos ^{-1}[\varepsilon+(1-\varepsilon) \cos \psi] \text {. }
$$

We compute

$$
\begin{array}{cr}
\sqrt{1-\varepsilon}<\frac{\sin \hat{\psi}}{\sin \psi}=\sqrt{\frac{(1+\varepsilon)+(1-\varepsilon) \cos \psi}{1+\cos \varepsilon}} \sqrt{1-\varepsilon}<\sqrt{1-\varepsilon^{2}} \\
\frac{d \hat{\psi}}{d \psi}=(1-\varepsilon) \frac{\sin \psi}{\sin \hat{\psi}}, & \hat{\psi}(0)=0
\end{array}
$$

and thus

$$
\sqrt{\frac{1-\varepsilon}{1+\varepsilon}} \psi<\hat{\psi}<\sqrt{1-\varepsilon} \psi .
$$

If $n=2$, we may set $\varepsilon=0$, cf. (103).

4. Uniqueness and existence

4.1. Symmetric solutions. We wish to show that for prescribed volume $V$ and contact angle $\gamma$ there is exactly one symmetric solution of (1). We note the symmetric solutions of (1) correspond biuniquely to the solutions of (9), which in turn are determined by the initial value $u_{0}$. To show there is at most one solution it thus suffices to show

$$
\left.\dot{V}=\frac{\partial V}{\partial u_{0}}\right]_{\psi}<0
$$


for each $u_{0}$ and (fixed) $\psi$ in $0<\psi \leqq \pi$. We have as before

$$
V=\sigma_{n}\left(r^{n} u-\frac{n}{n-1} r^{n-1} \sin \psi\right)
$$

and thus ${ }^{9}$

$$
\dot{V}=\sigma_{n} r^{n-2}\left(n \Delta \dot{r}+r^{2} \dot{u}\right)
$$

where $\sigma_{n}$ is the volume of the unit $n$-ball. Also

$$
\begin{aligned}
& \frac{\partial V}{\partial \psi}=\frac{\sigma_{n}}{n-1} \frac{r^{n+1} \sin \psi}{\Delta} \\
& \frac{\partial \dot{V}}{\partial \psi}=\frac{\sigma_{n} r^{n} \sin \psi}{\Delta^{2}}\left\{(n \Delta-\sin \psi) \dot{r}-r^{2} \dot{u}\right\} .
\end{aligned}
$$

We show $\dot{r}<0, \dot{u}>0$. We obtain from (9)

$$
\begin{aligned}
& \frac{d \dot{r}}{d \psi}=-\frac{r^{2} \dot{u}+\dot{r} \sin \psi}{(n-1) \Delta^{2}} \cos \psi \\
& \frac{d \dot{u}}{d \psi}=-\frac{r^{2} \dot{u}+\dot{r} \sin \psi}{(n-1) \Delta^{2}} \sin \psi
\end{aligned}
$$

with

$$
\dot{r}(0)=0, \quad \dot{u}(0)=1 .
$$

For $0<r<R$ we have

$$
r^{n-1} \sin \psi=(n-1) \int_{0}^{r} \rho^{n-1} u d \rho
$$

and writing $u=u\left(\rho ; u_{0}\right)=u\left(\rho\left(\downarrow ; u_{0}\right) ; u_{0}\right)$,

$$
\left.\left.\left.\left.\frac{\partial u}{\partial u_{0}}\right]_{\psi}=\frac{\partial u}{\partial \rho}\right]_{u_{0}} \dot{\rho}+\frac{\partial u}{\partial u_{0}}\right]_{\rho}=\dot{\rho} \tan \psi+\frac{\partial u}{\partial u_{0}}\right]_{\rho},
$$

we find from (120)

$$
-\dot{r} \Delta=r^{2-n} \int_{0}^{r} \rho^{n-1}(\dot{u}-\dot{\rho} \tan \psi) d \rho,
$$

and we see from $(119,120)$ that for each $u_{0}>0$ there is an initial interval $\mathscr{I}: 0<\boldsymbol{r}<\delta \leqq \pi$, in which $\dot{r}<0$.

In $\mathscr{I}$, we have by (118)

$$
\frac{d \dot{u}}{d \psi}>-\frac{r^{2} \dot{u} \sin \psi}{(n-1) \Delta^{2}}>-(n-1) \frac{r^{2}}{\sin \psi} \dot{u}
$$

${ }^{9}$ It is a formal (and not difficult) matter to prove the existence and requisite continuity properties of the derivatives in $u_{0}$. 
and hence

$$
\dot{u}>\exp \left\{-(n-1) \int_{0}^{\psi} \frac{\rho^{2}(\psi)}{\sin \psi} d \psi\right\}
$$

thus $\dot{u}>0$ in $\mathscr{I}$, and it follows from (116) that $d \dot{V} / d \psi<0$ in $\mathscr{F}$.

Let $\hat{\psi}$ be the smallest positive ir for which $\dot{r}=0$. If there is no such is or if $\hat{\psi} \geqq \pi$, then $d \dot{V} / d \psi<0$ in $0<\psi<\pi$, hence since $\dot{V}(0)=0$, there holds $\dot{V}<0$ in $0<\hat{\psi} \leqq \pi$. If $\hat{\psi}<\pi$ we have as above $\dot{V}(\hat{\psi})<0, \dot{u}(\hat{\psi})>0$, and also from (114)

$$
\dot{V}(\hat{\psi})=n \sigma_{n} r^{n}(\hat{\psi}) \dot{u}(\hat{\psi})>0 .
$$

This contradiction establishes that $\dot{r}<0$ on $0<\psi^{\prime}<\pi$, hence $d \dot{V} / d \dot{\psi}<0$ on that interval, hence $\dot{V}<0$ on $0<\dot{\psi} \leqq \pi$, for any choice of $u_{0}>0$. Since $u_{0}$ is arbitrary, the result yields the uniqueness of the symmetric solutions in the class of symmetric surfaces.

It remains to show there is at least one solution. To do so, we note that from (113), (6a), (28) follows $\lim _{u_{0} \rightarrow \infty} V(\psi)=0$, while from (113), (29), (40) follows $\lim _{u_{0} \rightarrow 0} V(\psi)=\infty$, for any fixed $\psi$ in $0<\psi \leqq \pi$. Thus, given $\psi=\gamma$, and any prescribed $V$, there exists at least one value $u_{0}$ at which $V$ is achieved.

4.2. General configurations. In the case of constant boundary angle $\gamma \leqq \pi / 2$, the symmetric solution $u(r)$ is unique in the class of all nonparametric solutions $v(x)$ of $(1)$ which bound the same volume, which meet the plane $u=0$ in a simple closed curve $\Gamma$ of class $C^{(3)}$, and which make with that plane the angle $\gamma$ at points of $\Gamma$.

Referring to $\S 4.1$, we see that it suffices to prove the surface $v(x)$ is symmetric. We note the associated solution $\hat{v}(x)$ of (3) has constant height on the image $\hat{\Gamma}$ of $\Gamma$, and the surface meets the vertical cylinder through $\hat{\Gamma}$ in the constant angle $\pi / 2-\gamma$. The symmetry of $\hat{v}$, and hence of $v$, can now be inferred from the theorem of Serrin [13].

5. The envelope. The family of solutions of (11) parametrized by $u_{0}$ admits an envelope, determined by the condition

$$
\left|\begin{array}{ll}
u^{\prime}(\psi) & r^{\prime}(\psi) \\
\dot{u}(\psi) & \dot{r}(\psi)
\end{array}\right|=0
$$

or, equivalently,

$$
F(\psi) \equiv \dot{u} \cos \psi-\dot{r} \sin \psi=0 .
$$




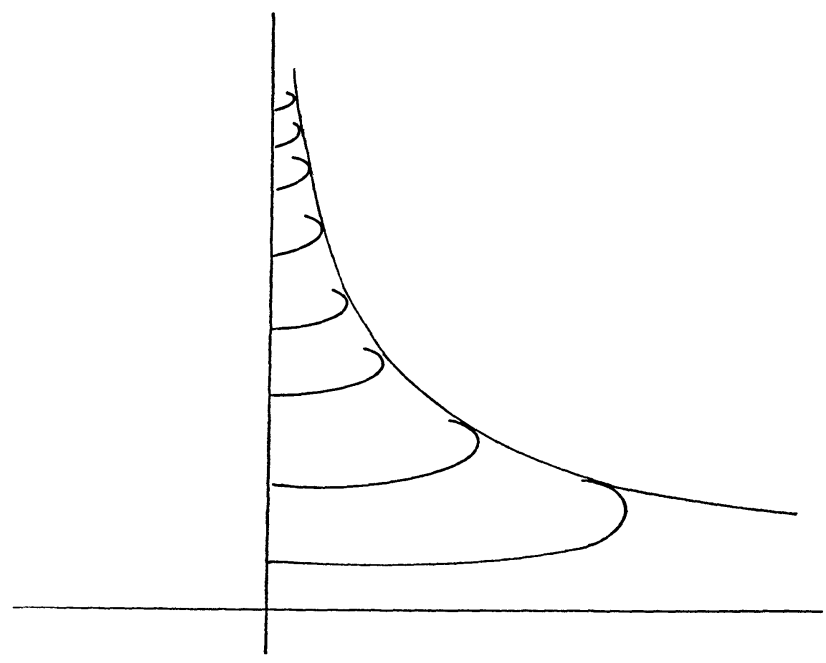

Figure 5. The envelope

Since, as shown in $\S 4.1, \dot{u}>0, \dot{r}<0$ on $0<\psi<\pi$, there can be no envelope points on $0<\psi \leqq \pi / 2$. We have $F(\pi / 2)>0, F(\pi) \leqq 0$, $F^{\prime}(\psi)=-\dot{u} \sin \psi-\dot{r} \cos \psi<0$ on $\pi / 2 \leqq \psi<\pi$. Thus on each integral curve there is exactly one contact point $\psi^{e}$ with the envelope, with $\pi / 2<\psi^{e}<\pi$. The envelope is illustrated in Figure 5.

6. An inclusion property. Referring again to the physical situation of symmetric drops on a planar surface, we normalize them to have a common axis of symmetry, and ask whether a given drop contains in its interior every drop of smaller volume that meets the plane with the same angle $\gamma$. In general this is not the case, as we see from the computer calculations in [3] (see also Figure 7). We shall show, however, that if $0<\gamma \leqq \pi / 2$, then the assertion holds whenever the ratio $V / \sin \psi$ is small enough and we shall give an explicit estimate of how small it must be.

We parametrize the solutions once more by $u_{0}$, and consider solutions $u=u\left(r ; u_{0}\right)$ and $u^{\delta}=u\left(r ; u_{0}+\delta\right)$ of $(4), \delta>0$. Let $v^{\delta}\left(r ; u_{0}\right)=u^{\delta}-\delta$, then $v^{\delta}\left(0 ; u_{0}\right)=u\left(0 ; u_{0}\right)$ and

$$
\begin{aligned}
& \left(r^{n-1} \sin \psi^{\delta}\right)_{r}=(n-1) r^{n-1} u^{\delta} \\
& \left(r^{n-1} \sin \psi\right)_{r}=(n-1) r^{n-1} u
\end{aligned}
$$

where $\psi^{\delta}$ is the angle $\psi$ measured on the curve $v^{\delta}$. Thus,

$$
r^{n-1}\left(\sin \psi^{\delta}-\sin \psi\right)=(n-1) \int_{0}^{r} \rho^{n-1}\left(u^{\delta}-u\right) d \rho
$$

from which we conclude $u^{\delta}-u$ is increasing in $r$. It follows that if the curve $u^{\delta}$ is moved rigidly downward a distance $\delta$, it will lie 
above the curve $u$ except at the single point of contact at $r=0$. To show the inclusion property corresponding to an angle $\gamma$, it thus suffices to show that for any $\delta>0$ there holds $v^{\delta}<u$ at the points where the angle $\gamma$ is achieved, and to this purpose it will suffice to know $d \dot{u} / d \psi<0$ in the interval $0<\psi<\gamma$, for all $u_{0}$ exceeding the given one. We proceed to determine sufficient conditions for that inequality; we first observe that, by (6), (40)

$$
\Delta \sim \frac{1}{n} r u_{0}
$$
as $r \rightarrow 0$

and thus, by $(119,122)$

$$
\dot{r} \sim-\frac{1}{u_{0}} r
$$

Writing (122) in the form

(131) $f(\psi) \equiv \dot{r} \sin \psi+r^{2} \dot{u}=\dot{r} r u+r^{2} \dot{u}+r^{2-n} \int_{0}^{r} \rho^{n-1}(\dot{u}-\dot{\rho} \tan \psi) d \rho$

and using $u(r) \rightarrow u_{0}, \quad \dot{u}(r) \rightarrow 1, \quad \dot{\rho}(r) \rightarrow 0$, we find $f(\psi) \sim r^{2} / n$; thus there is an interval $\mathscr{T}_{\dot{\delta}}$ : $0<\psi<\delta$, in which, by (118), $d \dot{u} / d \psi<0$.

At a first zero, $\hat{\psi}$, of $d \dot{u} / d \psi$, there would hold $f(\hat{\psi})=0$, $f^{\prime}(\hat{\psi}) \leqq 0$. We calculate

$$
f^{\prime}(\hat{\psi})=\frac{\dot{r} \cos \psi}{(n-1) \Delta}[(n-1) r u-(n+1) \sin \psi] .
$$

Since, as was shown in $\S 4.1, \dot{r}<0$, the inclusion property will follow on the interval $\mathscr{J}_{r}, 0<\gamma \leqq \pi / 2$, provided we can show

$$
g(\psi) \equiv(n-1) r u-(n+1) \sin \psi<0
$$

on $\mathscr{F}_{r}$, for the given surface and for all surfaces with smaller volume.

We show first that if, for given $\gamma, g(\psi)<0$ on $\mathscr{F}_{r}$ for one surface, then $g(\psi)<0$ on $\mathscr{I}_{r}$ for all surfaces with smaller volume. In view of the result of $\S 4.1$, it will suffice to show $\dot{g}(\psi)<0$ for each $\psi \in \mathscr{J}_{r}$.

We have $\dot{g}(\psi)=(n-1)(r \dot{u}+\dot{r} u)$. Since, as we have shown, $\dot{V}<0, \dot{r}<0$, it follows from $(114,10)$ that

$$
\dot{g}<-\frac{n-1}{r}\{(n-1) \Delta-\sin \psi\}<0,
$$

as was to be proved.

We now give the first form of the result of this section. 
Suppose $0<\gamma \leqq \pi / 2$, and

$$
u_{0}^{2}>\frac{2 n^{2}}{3(n-1)}(1-\cos \gamma) .
$$

Then every liquid drop with contact angle $\gamma$, corresponding to the initial value $\hat{u}_{0}>u_{0}$, can be translated rigidly so as to lie strictly interior to the drop corresponding to $u_{0}$.

Proof. It suffices to show that if $u_{0}$ satisfies (135), then $g\left(\psi ; u_{0}\right)<0$ on $\mathscr{F}_{r}$. From (135) and (6a) follow

$$
r^{2}<\frac{3}{2(n-1)}(1+\cos \gamma)
$$

From $(23,136)$ we find

$$
\begin{aligned}
(n-1) r u & <(n-1) \sin \gamma+(n-1)\left\{\frac{2 r^{2}}{n-1}(1-\cos \gamma)\right. \\
+ & \left.\frac{\sin ^{2} \gamma}{(n-1)^{2}}\right\}^{1 / 2}<(n-1) \sin \gamma+2 \sin \gamma=(n+1) \sin \gamma
\end{aligned}
$$

and thus $g\left(\gamma ; u_{0}\right)<0$. Since (135) obviously holds for all $\psi$ on $0 \leqq \psi<\gamma$, we conclude $g\left(\psi ; u_{0}\right)<0$ on $\mathscr{J}_{r}$, hence $d \dot{u} / d \psi<0$ on $\mathscr{F}_{r}$. Since (135) also holds for all larger $u_{0}$, the result follows.

From a physical point of view it is desirable to have the result in terms of the prescribed volume $V$. To do so, we use the lower bound (104) (or, if $n=2,(103)$ ) for $V$. Using also (28), we find that the choice

$$
\begin{aligned}
\frac{1}{\sigma_{n}} \frac{V}{\sin \gamma}< & \left(\frac{n}{n-1}\right)^{(n+1) / 2}\left(\frac{2(n+3)}{3}\right)^{-(n+1) / 2}(1+\cos \gamma)^{(n+1) / 2} \\
& \times \frac{1}{\sin ^{n+2} \gamma} \int_{0}^{\gamma} \sin ^{n+1} \theta d \theta
\end{aligned}
$$

ensures that the hypothesis (135) will be satisfied; thus, if $\gamma \leqq \pi / 2$, all drops of smaller volume can be strictly enclosed by any given one for which (138) holds.

In (138), $\hat{\gamma}=\gamma$ if $n=2$, otherwise $\hat{\gamma}$ is to be determined from (106) or (110). We note the essential qualitative content of (138) appears as a condition on the ratio $V / \sin \gamma$, rather than on volume alone.

7. Non-monotonicity. We complement the result of the preceding section by showing that for sufficiently large $\mathrm{V} / \mathrm{sin} \psi$, the 
inclusion property fails. To do so, we consider the height $q(\psi)=$ $u(\psi)-u_{0}$; we have by (76), (6a), that $\lim _{r \rightarrow \infty} u_{0}=0$, and hence, by (28), (29), (36), there exists

$$
q_{\infty}=\lim _{V \rightarrow \infty} q(\psi)=\sqrt{\frac{2}{n-1}(1-\cos \psi)}, \quad 0<\psi \leqq \pi .
$$

It will thus suffice to show that for all sufficiently large $V / \sin \psi$, there holds $q(\psi)>q_{\infty}$.

We suppose first $0<\psi \leqq \pi / 2$ and consider (33), from which

$$
u(\psi)>\sqrt{\frac{p+1}{p}} \sqrt{\frac{2(1-\cos \psi)}{n-1}} .
$$

We have

$$
\frac{p+1}{p}=1+\frac{1}{p}>1+\frac{n-1}{1+(r / k)} .
$$

By $(\mathbf{7 6}), \lim _{(\tau / \sin \psi) \rightarrow \infty} r(\psi)=\infty$, while

$$
\begin{aligned}
\sqrt{\frac{p+1}{p}} & >1+\frac{n-1}{2} \frac{1}{1+(r / k)}-\frac{1}{8}\left(\frac{n-1}{1+(r / k)}\right)^{2} \\
& >1+\frac{n-1}{2} \frac{k}{r}\left(1-\frac{k}{r}\right)-\frac{(n-1)^{2}}{8}\left(\frac{k}{r}\right)^{2}
\end{aligned}
$$

so that

$$
\sqrt{\frac{p+1}{p}}>1+\frac{n-1}{2} \frac{k}{r}-\frac{(n+3)(n-1)}{8}\left(\frac{k}{r}\right)^{2}
$$

for all $r>k$. Thus, using (31), (139-141),

$$
\begin{aligned}
q(\psi)=u(\psi)-u_{0} & >q_{\infty}+\left(\frac{1}{2 r}-\frac{u_{0}}{\sin \psi}\right) \sin \psi-\frac{(n+3)(n-1)}{8 r^{2}} \\
& \times \sqrt{\frac{1+\cos \psi}{2}} \sin \psi .
\end{aligned}
$$

By (63), (65) we have for all $r>s$

$$
\frac{u_{0}}{\sin \psi}<\frac{n}{n-1} \frac{1}{A_{0}} r^{(n-1) / 2} e^{-\sqrt{n-1} r} .
$$

Hence $q(\psi)>q_{\infty}$ for all sufficiently large $V / \sin \psi$, which was to be shown.

The case $\pi / 2<\psi \leqq \pi$ yields to an analogous reasoning, by adjoining the inequality (36) to (33). We suppress details. 


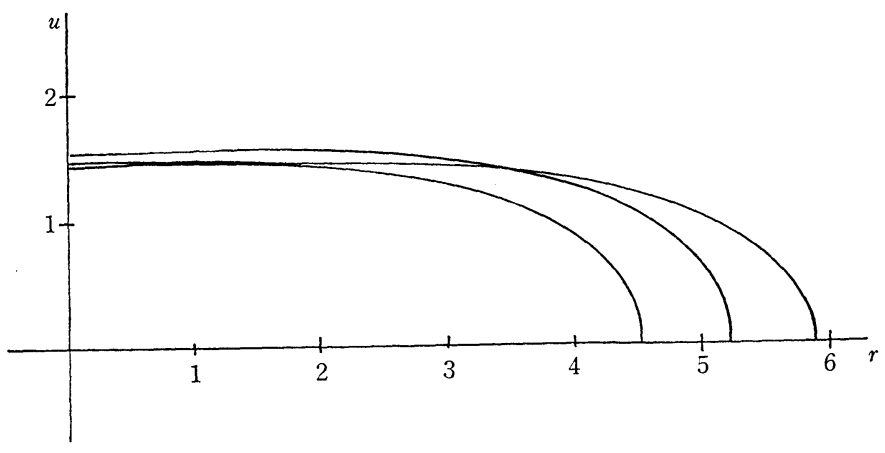

Figure 6. Non-monotonicity, $\gamma=\pi / 2$

Calculations to determine the maximum drop height $q_{M}$ and corresponding radius $r_{M}$ of wetted surface, for several choices of contact angle $\gamma$ in the case $n=2$, were performed by Heidi Bjørstad. The results, together with the (exactly known) limiting height $q_{\infty}$ (as $V \rightarrow \infty$ ) are summarized in Table 1. In Figure 6 some configurations near the extremal one are sketched for the case $\gamma=\pi / 2$.

\section{Unified estimates.}

8.1. Diameter II. We combine here some of the results of the preceding sections, in order to obtain estimates of the diameter of the wetted surface and of the drop, in terms of volume and contact angle.

Case 1. $0<\psi \leqq \pi / 2$ :

We have immediately from (76)

$$
r^{n}>\frac{(n-1) k}{\sigma_{n}} \frac{V}{\sin \psi}
$$

which provides a useful estimate when $V / \sin \psi$ is large. In the remaining case, (85) and (40) yield the estimate

$$
\left(\frac{2 r e^{\sqrt{1+(r / k)^{2}}-1}}{1+\sqrt{1+(r / k)^{2}}}\right)^{n+1}>\frac{\sin ^{n+2} \psi}{\sigma_{n} \int_{0}^{\psi} \sin ^{n+1} \theta d \theta} \frac{V}{\sin \psi} .
$$

Both estimates are universally valid, and are asymptotically exact for, respectively, large or small $(V / \sin \psi)$. For sufficiently small $(V / \sin \psi)$ we obtain additionally from $(90)$ the estimate

$$
r^{n+1}>\left(\frac{2-\varepsilon}{2}\right)^{n+1} \frac{1}{\sigma_{n}} \frac{V}{\psi-\frac{n+1}{n+2} \sin \psi}
$$

which is again asymptotically exact. 
To bound $r$ above, we consider again at first the case of large $V / \sin \psi$. We place (33) into (74), neglecting the explicit terms in $u_{0}$, to obtain

$$
r^{n}\left(\sqrt{\frac{p+1}{p}}-\frac{k n}{r}\right)<\frac{n-1}{\sigma_{n}} k \frac{V}{\sin \psi} .
$$

Using (141) and setting

$$
p^{n}=\frac{n-1}{\sigma_{n}} k \frac{V}{\sin \psi},
$$

we find immediately from (144)

$$
r^{n}<\frac{P^{n}}{\left(1-\frac{n+1}{2} \frac{k}{P}-\frac{(n+3)(n-1)}{8} \frac{k^{2}}{P^{2}}\right)}
$$

whenever the denominator is positive.

Putting (144) and (149) together, we have for any $\varepsilon>0$ and all sufficiently large $V / \sin \psi$,

$$
1<\frac{\sigma_{n}}{n-1} \frac{r^{n} \sin \psi}{k V}<1+\frac{k(n+1)}{(2-\varepsilon) P} .
$$

The left-hand inequality holds for all $V>0$, but yields a good estimate only when $V / \sin \psi$ is large. To study the remaining cases, we may start with the lower bound (104) for the volume, and estimate $u$ by (22) or (23). If

$$
\frac{r^{2} u_{0}^{2}}{\sin ^{2} \psi}<\frac{n}{n-1}
$$

we may use (22) to obtain

$$
u_{\psi}<\frac{\sin \psi}{r}\left(1+\frac{1}{n-1} \frac{r}{k}\right)
$$

from which, by (104), (92),

$$
\frac{V}{\sin \psi}>a_{n} \frac{r^{n+1}}{\sin ^{n+2} \psi}\left(\frac{n}{n-1+(r / k)}\right)^{n+1} \text {. }
$$

We have by (105) and the discussion following (85)

$$
a_{n}=\sigma_{n} \int_{0}^{\hat{\psi}} \sin ^{n+1} \theta d \theta>\frac{\sigma_{n}}{n+2} \sin ^{n+2} \hat{\psi} ;
$$

thus 


$$
\frac{V}{\sin \psi}>\frac{\sigma_{n}}{n+2} \frac{\sin ^{n+2} \hat{\psi}}{\sin ^{n+2} \psi} r^{n+1}\left(\frac{n}{n-1+(r / k)}\right)^{n+1} \text {. }
$$

Using (106) we find easily

$$
\frac{\sin \hat{\psi}}{\sin \psi}=\frac{2}{n} \sqrt{\frac{n-1+\cos \psi}{1+\cos \psi}}
$$

Setting

$$
Q^{n+1}=\frac{n+2}{\sigma_{n}} \frac{n}{2} \sqrt{\frac{1+\cos \psi}{n-1+\cos \psi}} \frac{1}{(k n)^{n+1}} \frac{V}{\sin \psi},
$$

we obtain

$$
r<(n-1) k \frac{Q}{1-Q}
$$

which holds whenever (151) holds. This result provides an estimate suitable for an "intermediate" range of $V / \sin \psi$.

The result could of course be strengthened by using the original definition for $a_{n}$ instead of the approximation (154), cf. (103) for even $n$.

We note the inequality

$$
\frac{n}{2} \sqrt{\frac{1+\cos \psi}{(n-1)+\cos \psi}}<\sqrt{\frac{n}{2}}
$$

provides a convenient simplification in (158).

For small values of $V / \sin \psi$ we write $(23)$ in the form

$$
\begin{aligned}
u_{\psi} & <\frac{\sin \psi}{r}\left(1+\frac{1}{n-1} \sqrt{1+\frac{r^{2}}{k^{2}}}\right) \\
& <\frac{n}{n-1} \frac{\sin \psi}{r}\left(1+\frac{1}{2 n} \frac{r^{2}}{k^{2}}\right) .
\end{aligned}
$$

The procedure above now yields

$$
r<k \sqrt{2 n} \frac{1-\sqrt{1-4 \hat{Q}^{2}}}{2 \hat{Q}}
$$

with

$$
\hat{Q}^{n+1}=\left(\frac{1}{k \sqrt{2 n}}\right)^{n+1} \frac{\sin ^{n+2} \psi}{\int_{0}^{\hat{\psi}} \sin ^{n+1} \theta d \theta} \frac{V}{\sigma_{n} \sin \psi}
$$

whenever $\hat{Q}<1 / 4$. Here $\hat{\psi}$ is determined by (110) and satisfies the estimates $(111,112)$. If $n=2$, we may set $\varepsilon=0$, cf. (103).

We have also the simpler, if slightly less precise, expression 
(163)

$$
r<k \sqrt{2 n}\left(\hat{Q}+4 \hat{Q}^{3}\right) .
$$

This result is in asymptotic agreement with $(145,146)$.

Case 2. $\pi / 2<\psi \leqq \pi$ :

From (18) we have

$$
r>R \sin \psi, \quad R=r\left(\frac{\pi}{2}\right) .
$$

We seek to estimate $R$, using the results for Case 1 , with $\psi=\pi / 2$. To do so we need an estimate for $V(\pi / 2)$ in terms of (the given) $V(\psi)$.

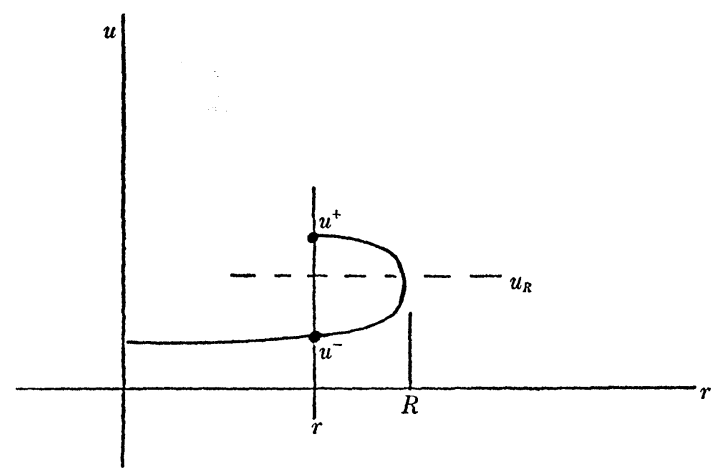

Figure 7. Proof of (168)

We integrate (4) twice along the common segment $[r, R]$, using, respectively, values $u^{+}, \psi^{+}$, or $u^{-}, \psi^{-}$on upper or lower parts of the solution curve (Figure 7 ). We find

$$
\begin{aligned}
R^{n-1}-r^{n-1} \sin \psi^{+} & =(n-1) \int_{r}^{R} \rho^{n-1} u^{+} d \rho \\
R^{n-1}-r^{n-1} \sin \psi^{-} & =(n-1) \int_{r}^{R} \rho^{n-1} u^{-} d \rho .
\end{aligned}
$$

Since $u^{+}>u^{-}$, we find by subtracting the second equation from the first

$$
\sin \psi^{+}<\sin \psi^{-}
$$

and thus

$$
u^{+}-u_{R}<u_{R}-u^{-} .
$$

We conclude immediately from (74)

$$
2 V\left(\frac{\pi}{2}\right)>V\left(\psi^{+}\right)+V\left(\psi^{-}\right)>V(\psi)
$$

where we have replaced $\psi^{+}$by $\psi^{\prime}$. 
For large values of $V$ we thus find from (144)

$$
R^{n}>\frac{\sqrt{n-1}}{2 \sqrt{2} \sigma_{n}} V
$$

while from (145) we find

$(\underline{\mathbf{1 7 0}})$

$$
\left(\frac{2 R e^{\sqrt{1+2(n-1) R^{2}}-1}}{1+\sqrt{1+2(n-1) R^{2}}}\right)^{n+1}>\frac{1}{\sigma_{n} \sqrt{\pi}} \frac{\Gamma\left(\frac{n+3}{2}\right)}{\Gamma\left(\frac{n}{2}+1\right)} V
$$

which is preferable for small $V$. These estimates together with (164) yield a lower bound on $r$ in the range of $\psi$ considered.

If

$$
R>\frac{n}{\sqrt{2(n-1)}} \frac{1-\sin ^{n+1} \psi}{1-\sin ^{n} \psi},
$$

the estimate (cf. (53))

$$
r^{n}>R^{n} \frac{\frac{\sqrt{2(n-1)}}{n} R-1}{\frac{\sqrt{2(n-1)}}{n} R-\sin \psi}
$$

is preferable to (164). We now find from (169) the result

$$
r_{T}>\left(\frac{n-1}{8}\right)^{1 / 2 n}\left(\frac{1}{\sigma_{n}} V\right)^{1 / n}-\frac{1}{\sqrt{2(n-1)}}
$$

for all sufficiently large $V$.

To bound $r$ above in this case, we observe first that $V(\pi / 2)<$ $V(\psi)$ if $\psi>\pi / 2$, hence (149) yields the estimate, for large $V$,

$$
R^{n}<\frac{P_{R}^{n}}{\left(1-\frac{n+1}{2 \sqrt{n-1}} \frac{1}{P_{R}}-\frac{n+3}{16} \frac{1}{P_{R}^{2}}\right)}
$$

with

$$
P_{R}^{n}=\frac{1}{\sigma_{n}} \sqrt{\frac{n-1}{2}} V .
$$

The procedure leading to (158) yields now

$$
R<\sqrt{\frac{n-1}{2}} \frac{Q_{R}}{1-Q_{R}}
$$

with 


$$
Q_{R}^{n+1}=\frac{n+2}{\sqrt{2} \sigma_{n}}\left(\frac{\sqrt{2(n-1)}}{n}\right)^{n} V
$$

and (163) becomes

$$
R<\sqrt{\frac{n}{n-1}}\left(\hat{Q}_{R}+4 \hat{Q}_{R}^{3}\right)
$$

with

$$
\widehat{Q}_{R}^{n+1}=\frac{n+2}{\sigma_{n}} \frac{\sqrt{n}}{2}\left(\frac{n-1}{n}\right)^{n / 2} V .
$$

Similarly, analogues of (161), (163) can be given for small drops. An upper bound for $r$ now follows from (69) or (71). We find in particular from (174), (73),

$$
r_{T}<\left(\frac{n-1}{2}\right)^{1 / 2 n}\left(\frac{1}{\sigma_{n}} V\right)^{1 / n}-\frac{1}{2 \sqrt{n-1}}
$$

for all sufficiently large $V$ (compare (173)).

8.2. Height II. We reformulate here the results of $\S 3.2$ in terms of the (physical) drop height

$$
q(\psi)=u(\psi)-u_{0}
$$

and, for the case is $>\pi / 2$, the "semi-height"

$$
h=u_{R}-n_{0}
$$

from the level of maximum diameter to the vertex height, which is useful in some applications (cf. Roberts [12] and the references cited there).

We find from (33)

$$
\begin{aligned}
\frac{q(\psi)}{\sin \psi}> & \frac{u_{0}}{\sin \psi}\left\{\sqrt { \frac { p + 1 } { p } } \left[\frac{1}{k^{2}(n-1)^{2}} \frac{\sin ^{2} \psi}{u_{0}^{2}}\right.\right. \\
& \left.\left.+\frac{p(n-1)^{2}+1}{n^{2}}\right]^{1 / 2}-1\right\}, \quad 0<\psi \leqq \pi / 2 .
\end{aligned}
$$

The right side of (181) is an increasing function of $\sin \psi / u_{0}$; using (6a) we see that it is decreasing in $p$. If $V / \sin \psi$ is large, we may estimate $p$ using (147) et seq., and $\sin \psi / u_{0}$ using (63). If $\mathrm{V} / \mathrm{sin}$ is is small, we may use (6a) to convert (181) into

$$
\begin{aligned}
& \frac{q(\psi)}{\sin \psi}>\frac{1}{n} \frac{u_{0}}{\sin \psi}\left(\sqrt{1+\left(\frac{n}{n-1}\right)^{2} \frac{1}{k_{k}^{2}} \frac{\sin ^{2} \psi}{u_{0}^{2}}}-1\right), 0<\psi \leqq \pi / 2 \\
& 0<\%
\end{aligned}
$$


and $\sin \psi / u_{0}$ can be estimated using (85) et seq. Both formulas are correct in all cases, and we obtain a bound of the form

$$
\frac{q(\psi)}{\sin \psi}>F\left(\frac{V}{\sigma_{n} \sin \psi} ; k\right), \quad 0<\psi \leqq \pi / 2,
$$

with the asymptotic properties

(모)

$$
\begin{array}{cr}
F(t, k) \sim \frac{1}{(n-1)} \frac{1}{2 k^{2}}\left(\frac{\sin ^{(n+2)} \psi}{\int_{0}^{\psi} \sin ^{(n+1)} \theta d \theta}\right)^{1 /(n+1)} t^{1 /(n+1)}, & t \rightarrow 0 \\
F(t, k) \sim \frac{1}{k(n-1)}+\frac{1}{2}\left(\frac{1}{k(n-1)}\right)^{1 / n} t^{-1 / n}, & t \rightarrow \infty .
\end{array}
$$

Setting $\psi=\pi / 2$, we obtain from (181), (182)

$$
\begin{gathered}
h>u_{0}\left\{\sqrt{\frac{1+p}{p}} \sqrt{\frac{2}{n-1} \frac{1}{u_{0}^{2}}+\frac{1+p(n-1)^{2}}{n^{2}}}-1\right\} \\
h>\frac{1}{n} u_{0}\left(\sqrt{1+\frac{2 n^{2}}{n-1} \frac{1}{u_{0}^{2}}-1}\right),
\end{gathered}
$$

the former relation being more precise, the latter more convenient. From (184), (185) we find

$$
h>H\left(\frac{1}{\sigma_{n}} V\right)
$$

where, asymptotically

$$
H(t) \sim\left(\frac{2}{\sqrt{\pi}} \frac{\Gamma\left(\frac{n+3}{2}\right)}{\Gamma\left(\frac{n+2}{2}\right)}\right)^{1 /(n+1)} t^{1 /(n+1)}, \quad t \rightarrow 0
$$

$$
H(t) \sim \sqrt{\frac{2}{n-1}}+2^{(1-n) / n} t^{-(1 / n)},
$$

If $\psi>\pi / 2$, we write

$$
q(\psi)=u(\psi)-u_{R}+h
$$

From (36)

$$
u(\psi)-u_{R}>u_{R}\left(\sqrt{1-\frac{2}{n-1} \frac{\cos \psi}{u_{R}^{2}}}-1\right)
$$

and from (22)

$$
u_{R}<\frac{1}{R}+\sqrt{\frac{2}{n-1}-\frac{1}{n-1} \frac{1}{R^{2}}+\frac{1}{n} u_{0}^{2}} .
$$


By (144),

$$
R>\left(\sqrt{\frac{n-1}{2}} \frac{1}{\sigma_{n}} V\right)^{1 / n} .
$$

We may thus apply (63) whenever

$$
\left(\sqrt{\frac{n-1}{2}} \frac{1}{n} V\right)^{1 / n}>\frac{1}{2} \frac{n+1}{\sqrt{n-1}} .
$$

For $V$ satisfying (195) we apply (63) and the estimates (174) et seq. to obtain bounds below for $u\left(\psi^{\prime}\right)-u_{R}$ of the form

$$
u(\psi)-u_{n}>G\left(\frac{1}{\sigma_{n}} V ; \psi\right), \quad \frac{\pi}{2}<\psi \leqq \pi,
$$

where, asymptotically,

$$
\begin{aligned}
G(t ; \psi) & \sim-\sqrt{\frac{2}{n-1}} \frac{\cos \psi}{1+\sqrt{1-\cos \psi}}+\left(\frac{2}{n-1}\right)^{1 / 2 n} \\
& \times \frac{\cos \psi}{\sqrt{1-\cos \psi}(1+\sqrt{1-\cos \psi})} t^{-1 / n},
\end{aligned}
$$

To include the case of $V$ that do not satisfy (195) we note from (23)

$$
u_{R}<\frac{1}{R}+\sqrt{\frac{2}{n-1}+\frac{1}{(n-1)^{2}} \frac{1}{R^{2}}} .
$$

Applying (145) with $\psi=\pi / 2$, we are led again to a relation of the form (196), with

(199)

$$
\begin{aligned}
& G(t ; \psi) \sim-\frac{1}{n}\left(\frac{2}{\sqrt{\pi}} \frac{\Gamma\left(\frac{n+3}{2}\right)}{\Gamma\left(\frac{n+2}{2}\right)}\right)^{1 /(n+1)} t^{1 /(n+1)} \cos \psi \\
& \quad+\frac{(n-1)(n+2)}{n^{2}}\left(\frac{2}{\sqrt{\pi}} \frac{\Gamma\left(\frac{n+3}{2}\right)}{\Gamma\left(\frac{n+2}{2}\right)}\right)^{3 /(n+1)} t^{3 /(n+1)} \cos \psi, t \rightarrow 0 .
\end{aligned}
$$

We turn our attention now to the question of upper bounds. From (22) we find

$$
\begin{aligned}
& \frac{q(\psi)}{\sin \psi}<\frac{1}{\gamma}+\frac{u_{0}}{\sin \psi}\left\{\left[\frac{1}{(n-1)^{2} k^{2}} \frac{\sin ^{2} \psi}{u_{0}^{2}}\right.\right. \\
& \left.\left.-\frac{1}{n-1} \frac{1}{r^{2}} \frac{\sin ^{2} \psi}{u_{0}^{2}}+\frac{1}{n}\right]^{1 / 2}-1\right\}, \\
& 0<\psi^{\prime} \leqq \frac{\pi}{2} .
\end{aligned}
$$


With the aid of $(28),(6 a, b)$ we find easily that the right side of (200) is decreasing in $r$, increasing in $\sin \psi / u_{0}$. The inequalities (40), (144) thus yield, in all cases, an estimate of the form

$$
\frac{q(\psi)}{\sin \psi}<L\left(\frac{V}{\sigma_{n} \sin \psi} ; k\right), \quad 0<\psi \leqq \frac{\pi}{2},
$$

with

$$
\begin{array}{rrr}
L(t ; k) \sim \frac{1}{(n-1)} \frac{1}{2 k^{2}}\left(\frac{\sin ^{(n+2)} \psi}{\int_{0}^{\psi} \sin ^{(n+1)} \theta d \theta}\right)^{1 /(n+1)} t^{1 /(n+1)}, & t \rightarrow 0 \\
& L(t ; k) \sim \frac{1}{k(n-1)}+\left(\frac{1}{(n-1) k}\right)^{1 / n} t^{-1 / n}, & t \rightarrow \infty .
\end{array}
$$

These results should be compared with (184), (185). We note $q / \sin \psi$ is bounded from zero and from infinity, depending (essentially) only on $V / \sin \psi$. Asymptotically, the upper and lower bounds move together as $\psi$ changes, but vary at most by a factor equal to two, for small $t$, and equal to $\sqrt{2}$ for large $t$.

Setting $\psi=\pi / 2$ we obtain

$$
h<M\left(\frac{1}{\sigma_{n}} V\right)
$$

with

$$
M \sim\left(\frac{2}{\sqrt{\pi}} \frac{\Gamma\left(\frac{n+3}{2}\right)}{\Gamma\left(\frac{n+2}{2}\right)}\right)^{1 /(n+1)} t^{1 / n+1)}, \quad t \rightarrow 0
$$

$$
M \sim \sqrt{\frac{2}{n-1}}+2^{1 / n} t^{-1 / n},
$$

in agreement with $(\mathbf{1 8 9}, \mathbf{1 9 0})$.

If $\psi>\pi / 2$, we first note from (28), (40), (174) et seq., and the observation $V(\pi / 2)<V(\psi)$, that there is a relation of the form

$$
q(\psi)<N\left(\frac{1}{\sigma_{n}} V ; \psi\right)
$$

From (25), (144), we obtain immediately that $N(t ; \psi)$ can be chosen to satisfy

$$
N(t ; \psi) \sim \sqrt{\frac{2(1-\cos \psi)}{n-1}}+\left(\frac{1}{(n-1) k}\right)^{1 / n} t^{-1 / n}, \quad t \rightarrow \infty,
$$


in agreement with $(\mathbf{1 9 0}, 198)$. From $(28),(40)$ we see that we can have

(209)

$$
N(t ; \psi) \sim(1-\cos \psi)\left(\frac{2}{\sqrt{\pi}} \frac{\Gamma\left(\frac{n+3}{2}\right)}{\Gamma\left(\frac{n+2}{2}\right)}\right)^{1 /(n+1)} t^{1 /(n+1)}, \quad t \rightarrow 0,
$$

a result in agreement with $(\mathbf{1 8 9}, \mathbf{1 9 9})$.

All the asymptotic estimates of this section can be written as explicit inequalities, by using the estimates from which they are derived. We do so for some particular situations in the following section, where we compare some of our estimates with those of other authors.

8.3. Height III. Laplace [10, pp. 971-993] considered a drop of mercury resting on a glass surface, the diameter of whose wetted circle is $10 \mathrm{~cm}$. For this configuration he calculated, using-essentially-a method of matching expansions, a drop height $q=$ $0.3397 \mathrm{~cm}$. As is characteristic of the method, no error bounds can be given. Laplace chose the example to correspond with measurements due to Gay-Lussac, who obtained $q=0.3378$, and to Segner, who found $q=0.3407$.

Although the example does not correspond directly with the format of the present paper, we can adapt our results as follows:

Taking for $\kappa, \gamma$ the values $\kappa=400 / 13, \gamma=136.8^{\circ}$ used by Laplace, we replace $r, R$ in (53), (69) by $\sqrt{\kappa} r, \sqrt{\kappa} R$ and set $r=5$ to obtain (with $n=2$ )

$$
5.0305<R<5.0418
$$

For this range of $R$, there holds $R>s$; thus we may apply (63), (64) to obtain

$$
u_{0}<2.5 \times 10^{-11} .
$$

Using (186), and (200) with $\psi=\pi / 2$, we compute

$$
0.2582<h<0.2613 \text {. }
$$

According to (211), $u_{0}$ can be neglected, and we may set $u_{R} \approx h$ in (24), (36) to obtain effective bounds above and below for $u(\gamma)-u_{R}$. The monotonicity properties of those relations, in $h$ and in $R$, yield, in view of (210), (212),

$$
0.0788<u(\gamma)-u_{R}<0.0811 .
$$


Noting again that $u_{0}$ can be neglected, we set $u_{R} \approx h$ in (24), (36) to obtain directly from those estimates

$$
0.3377<q<0.3416
$$

a result that appears to substantiate both the experimental results and Laplace's calculation.

Laplace's values for $\kappa, \gamma$, derive from measurements cited earlier in his article. Current literature suggests a value $\kappa \sim 28.6$, which is somewhat less than Laplace's value. (Bashforth and Adams in [2] determine $\kappa \sim 40$.) There is poor agreement, even in modern literature, on the value for $\gamma$. If we use the values $\kappa=28.6, \gamma=$ $140^{\circ}$, as suggested in [1], then our calculations show $q>0.354$, which exceeds both Laplace's result and those of the two experiments he cites. Nevertheless, both Laplace's choice and that of Bashforth and Adams were likely appropriate to the materials that were studied; both surface tension and contact angle are sensitive to impurities in the materials, and techniques for purification of mercury have improved greatly in the ensuing years.

\section{The capillary tube.}

9.1. Equivalence. We restrict attention here to the case $0<\psi \leqq \pi / 2$. We have seen in $\S 2$ that the problems of the sessi: drop and that of the capillary tube in an infinite vessel transform formally into each other. Much of the foregoing analysis has been carried out in terms of the latter formulation, and the results (notably of $\S \S 3,4$ ) apply to that physical situation. Many of the results were most conveniently expressed in terms of the ratio $\sin \psi / u_{0}$, which has immediate physical significance for the capillary problem.

The physical interpretation of $u_{0}$ for the sessile drop is not immediately clear. We were, however, able to transform our results (and to derive some new ones) into formulations regarding the ratio $V / \sin \psi$, as seems appropriate for this problem; we note, however, that $V$ has no evident physical meaning for the capillary tube.

9.2. Reciprocity. The two above ratios are not independent of each other, but are connected by simple relations. Specifically, we note from (40) and (45)

$$
\frac{n}{n-1} \frac{\sin \psi}{u_{0}}<F(r ; k)
$$

the symbol $k$ denoting in this context an "inessential" dependence 
on $\psi$, that is, there exists $F^{*}(r)<\infty$ such that $F(r, k)<F^{*}(r)$ for all $\psi$ in $0<\psi \leqq \pi / 2$.

From $(6 a)$

$$
\frac{n}{n-1} \frac{\sin \psi}{u_{0}}>r
$$

and also from (63), whenever $r>s$,

$$
\frac{\sin \psi}{u_{0}}>\hat{F}(r ; k)
$$

which grows exponentially in $r$.

We have from (145)

$$
\frac{1}{\sin \psi} V(\psi)<\frac{\sigma_{n}}{\sin ^{n+2} \psi} \int_{0}^{\psi} \sin ^{n+1} \theta d \theta F^{n+1}(r ; k),
$$

or alternatively from (144)

$$
\frac{1}{\sin \psi} V(\psi)<\frac{\sigma_{n}}{k(n-1)} r^{n}
$$

especially for larger $r$. In the other direction we have from (149)

$$
\frac{(1+\varepsilon)}{\sin \psi} V(\psi)>\frac{\sigma_{n}}{k(n-1)} r^{n}
$$

for large $r$, and from (163)

$$
\frac{(1+\varepsilon)}{\sin \psi} V(\psi)>\frac{\sigma_{n}}{\sin ^{n+2} \psi} \int_{0}^{\hat{\psi}} \sin ^{n+1} \theta d \theta \cdot r^{n+1}
$$

for small $r$.

Putting these results together, we find a general inequality of the form

$$
G_{1}\left(\frac{\sin \psi}{u_{0}} ; k\right) \leqq \frac{V}{\sin \psi} \leqq G_{2}\left(\frac{\sin \psi}{u_{0}} ; k\right)
$$

Both functions $G_{1}(\tau ; k), G_{2}(\tau ; k)$ can be obtained explicitly from the indicated estimates. We may assume in what follows that a best possible choice has been made. We have, asymptotically for small values of the two ratios (or, equivalently, of $r$ ),

$$
G_{1}(\tau ; k), G_{2}(\tau ; k) \sim\left(\frac{n}{n-1}\right)^{n+1} \frac{\sigma_{n}}{\sin ^{n+2} \psi} \int_{0}^{\psi} \sin ^{n+1} \theta d \theta \cdot \tau^{n+1},
$$


We note these functions have identical asymptotic dependence on $k$, as $\tau \rightarrow 0$.

To obtain a corresponding asymptotic estimate for large $r$ we observe from $(45,63)$

$$
\frac{1}{r} \log \frac{\sin \psi}{u_{0}} \sim \sqrt{n-1},
$$

We thus find from (150), (222)

$$
G_{1}(\tau ; k), G_{2}(\tau ; k) \sim \frac{\sigma_{n}}{k}(n-1)^{-(n+2) / 2} \log ^{n} \tau, \quad \tau \rightarrow \infty .
$$

From (223), (225) we see that the two ratios are asymptotically determined in terms of each other, both for small and for large values of $r$.

Similar comments apply to the ratio $q / \sin \psi$, as one sees immediately from the relations (184-5), (202-3). The drop height $q$ has a physical meaning also for the capillary tube, as the height of the meniscus.

Collecting estimates in terms of wetting radius $r$, we find that for $r \rightarrow 0$ there holds

$$
\begin{aligned}
r^{-(n+1)} \frac{V}{\sigma_{n} \sin \psi} \sim \frac{1}{\sin ^{n+2} \psi} \int_{0}^{\psi} \sin ^{n+1} \theta d \theta \\
r^{-1} \frac{\sin \psi}{u_{0}} \sim \frac{n-1}{n} \\
r^{-1} \frac{q}{\sin \psi} \sim \frac{1}{1+\cos \psi}
\end{aligned}
$$

while for $r \rightarrow \infty$ we find

$(\underline{227})$

$$
\begin{gathered}
r^{-n} \frac{V}{\sigma_{n} \sin \psi} \sim(n-1) \sqrt{\frac{1+\cos \psi}{2(n-1)}} \\
\frac{1}{r} \log \frac{\sin \psi}{u_{0}} \sim \sqrt{n-1} \\
r\left(\frac{q}{\sin \psi}-\frac{1}{n-1} \sqrt{\frac{2(n-1)}{1+\cos \psi}}\right) \sim \lambda
\end{gathered}
$$

with $1 / 2 \leqq \lambda \leqq 1$.

These results are compared with numerical calculations for the case $n=2$, in Table 2. For convenience in engineering applications, the tabulations are given in terms of the (nondimensional) Bond number, referred to unit radius of wetted surface. The present variable $r$ (under our normalization $k=1$ ) is retrieved by the transformation $B=r^{2}$. 
Table 1. Parameters for drops of maximum height corresponding to varying contact angles.

\begin{tabular}{l|ccccc}
\hline \multicolumn{1}{c|}{$\gamma$} & $\pi / 2$ & $\pi / 3$ & \multicolumn{1}{c}{$\pi / 4$} & $\pi / 8$ & $\pi / 20$ \\
\cline { 2 - 6 }$p_{M}$ & 1.51333 & 1.07571 & 0.824911 & 0.421337 & 0.169539 \\
$q_{\infty}$ & 1.41421 & 1.00000 & 0.765367 & 0.390181 & 0.156918 \\
$q_{M} / q_{\infty}$ & 1.07009 & 1.07571 & 1.07780 & 1.07985 & 1.08043 \\
$r_{M}$ & 5.24325 & 5.41855 & 5.48874 & 5.56093 & 5.58218 \\
$V$ & 100.351 & 71.8385 & 55.3742 & 28.4631 & 11.4773 \\
$V / \sin \gamma$ & 100.351 & 82.953 & 78.311 & 74.378 & 73.368 \\
$\frac{V \sqrt{1+\cos \gamma}}{\sin \gamma}$ & 100.351 & 101.562 & 102.318 & 103.165 & 103.438 \\
\hline
\end{tabular}

Table 2a. Asymptotic properties, small B

\begin{tabular}{|c|c|c|c|c|c|}
\hline \multirow{2}{*}{$u_{0}$} & \multirow{2}{*}{$\sin \gamma$} & 3 & $(1+\cos \gamma)^{2}$ & 2 sin $\gamma$ & \multirow{2}{*}{$\frac{1+\cos \gamma}{r} \frac{q}{\sin \gamma}$} \\
\hline & & $\pi r^{3}$ & $2+\cos \gamma$ & & \\
\hline \multicolumn{6}{|l|}{$B=1$} \\
\hline 1.636 & 0.9478 & & 0.9007 & 1.158 & 0.8887 \\
\hline 1.455 & 0.8359 & & 0.9293 & 1.149 & 0.9122 \\
\hline 1.273 & 0.7276 & & 0.9415 & 1.143 & 0.9230 \\
\hline 1.091 & 0.6214 & & 0.9486 & 1.139 & 0.9296 \\
\hline 0.9091 & 0.5165 & & 0.9532 & 1.136 & 0.9340 \\
\hline 0.7273 & 0.4123 & & 0.9563 & 1.134 & 0.9370 \\
\hline 0.5455 & 0.3088 & & 0.9584 & 1.132 & 0.9391 \\
\hline 0.3636 & 0.2057 & & 0.9597 & 1.131 & 0.9405 \\
\hline 0.1818 & 0.1028 & & 0.9605 & 1.131 & 0.9413 \\
\hline \multicolumn{6}{|l|}{$B=.1$} \\
\hline 5.750 & 0.9231 & & 0.9892 & 1. 015 & 0.9878 \\
\hline 5.175 & 0.8301 & & 0.9920 & 1. 015 & 0.9901 \\
\hline 4.600 & 0.7375 & & 0.9933 & 1.014 & 0.9914 \\
\hline 4.025 & 0.6450 & & 0.9942 & 1.014 & 0.9922 \\
\hline 3.450 & 0.5527 & & 0.9949 & 1.013 & 0.9927 \\
\hline 2.875 & 0.4605 & & 0.9952 & 1. 013 & 0.9931 \\
\hline 2.300 & 0.3683 & & 0.9955 & 1. 013 & 0.9934 \\
\hline 1.725 & 0.2762 & & 0.9955 & 1. 013 & 0.9936 \\
\hline 1.150 & 0.1841 & & 0.9958 & 1.013 & 0.9937 \\
\hline 0.5750 & 0.09050 & & 0.9958 & 1. 013 & 0.9938 \\
\hline \multicolumn{6}{|l|}{$B=.01$} \\
\hline 18.18 & 0.9105 & & 0.9990 & 1.002 & 0.9988 \\
\hline 16.36 & 0.8194 & & 0.9992 & 1.001 & 0.9990 \\
\hline 14.55 & 0.7283 & & 0.9993 & 1.001 & 0.9991 \\
\hline 12.73 & 0.6372 & & 0.9994 & 1. 001 & 0.9992 \\
\hline 10.91 & 0.5462 & & 0.9995 & 1.001 & 0.9993 \\
\hline 9.091 & 0.4551 & & 0.9995 & 1.001 & 0.9993 \\
\hline 7.273 & 0.3641 & & 0.9995 & 1.001 & 0.9993 \\
\hline 5.455 & 0.2731 & & 0.9996 & 1. 001 & 0.9994 \\
\hline 3. 636 & 0.1820 & & 0.9995 & 1. 001 & 0.9994 \\
\hline \multirow{2}{*}{\multicolumn{6}{|c|}{$B=.001^{1.010}$}} \\
\hline & & & & & \\
\hline 57.50 & 0.9092 & & 0.9999 & 1.000 & 0.9999 \\
\hline 51.75 & 0.8183 & & 0.9999 & 1.000 & 0.9999 \\
\hline 46.00 & 0.7274 & & 0.9999 & 1.000 & 0.9999 \\
\hline 40.25 & 0.6364 & & $0.99,99$ & 1.000 & 0.9999 \\
\hline 34.50 & 0.5455 & & 0.9999 & 1.000 & 0.9999 \\
\hline 28.75 & 0.4546 & & 0.9999 & 1.000 & 0.9999 \\
\hline 23.00 & 0.3637 & & 0.9999 & 1.000 & 0.9999 \\
\hline 17.25 & 0.2728 & & 0.9999 & 1.000 & 0.9999 \\
\hline 11.50 & 0.1818 & & 0.9999 & 1.000 & 0.9999 \\
\hline 5.750 & 0.09092 & & 0.9999 & 1.000 & 0.9999 \\
\hline
\end{tabular}


Table 2b. Asymptotic properties, large B

\begin{tabular}{llccc}
\hline \multicolumn{1}{c}{$u_{0}$} & $\sin \gamma \frac{1}{\pi r^{2}} \sqrt{\frac{1+\cos \gamma}{2}} \frac{V}{\sin \gamma}$ & $\frac{1}{r} \log \frac{\sin \gamma}{u_{0}}$ & $r\left(\frac{q}{\sin \gamma}-\sqrt{\frac{2}{1+\cos \gamma}}\right)$ \\
\hline$B=10$ & 0.9027 & 0.6534 & 0.5014 & 0.05613 \\
0.1849 & 0.7015 & 0.6175 & 0.4922 & 0.02600 \\
0.1479 & 0.5171 & 0.6009 & 0.4768 & 0.01138 \\
0.1109 & 0.3411 & 0.5916 & 0.4834 & 0.002945 \\
0.07396 & 0.1695 & 0.5867 & 0.4815 & -0.001579 \\
0.03698 & 0.8946 & 0.8794 & 0.7954 & 0.5786 \\
$B=100$ & 0.6492 & 0.8646 & 0.7921 & 0.5528 \\
0.0003143 & 0.4251 & 0.8582 & 0.7903 & 0.5435 \\
0.0002358 & 0.2105 & 0.8551 & 0.7893 & 0.5394 \\
0.0001572 & 0.9636 & 0.9642 & 0.9184 & 0.5769 \\
0.00007859 & 0.9586 & 0.9174 & 0.5408 \\
$B=1000$ & 0.6926 & 0.9569 & 0.9170 & 0.5313 \\
$.2353 \times 10^{-12}$ & 0.946 & 0.9545 & 0.9165 & 0.5191 \\
$.2000 \times 10^{-12}$ & 0.7946 & 0.9161 & 0.5138 \\
$.1765 \times 10^{-12}$ & 0.695 & \\
$.1176 \times 10^{-12}$ & 0.4526 & 0.9533 & & \\
$.05882 \times 10^{-12}$ & 0.2240 & &
\end{tabular}

\section{REFERENCES}

1. A. W. Adamson, Physical Chemistry of Surfaces, 3rd ed., John Wiley \& Sons, 1976.

2. F. Bashforth and J. C. Adams, An Attempt to Test the Theories of Capillary Action, University Press, Cambridge, England, 1883.

3. P. Concus, Static menisci in a vertical right circular cylinder, J. Fluid Mech., 34 (1968), 481-495.

4. P. Concus and R. Finn, The shape of a pendent liquid drop, Philos. Trans. Roy. Soc. London Ser. A, 292 (1979), 307-340.

5. R. Finn, Some comparison properties and bounds for capillary surfaces, Complex Analysis and its Applications, Moscow Math. Soc., volume dedicated to I.N. Vekua, Scientific Press, Moscow, 1978.

6. E. Gonzalez, Sul problema della goccia appoggiata, Rend. Sem. Mat. Univ. Padova, 55(1976), 289-302.

7. - Regolarità per il problema della goccia appoggiata, Rend. Sem. Mat. Univ. Padova, to appear.

8. E. Gonzalez and I. Tamanini, Convessità della goccia appoggiata, Rend. Sem. Mat. Univ. Padova, to appear.

9. W. E. Johnson and L. M. Perko, Interior and exterior boundary value problems from the theory of the capillary tube, Arch. Rational Mech. Anal., 29 (1968), 125-143.

10. P. S. Laplace, Supplement to "Theory of Calillary Attraction", Supplement to vol. X of "Celestial Mechanics", translated by N. Bowditch, vol. IV, Chelsea Publishing Co., New York, 1966. (Originally published 1806).

11. Th. Lohnstein, Dissertation, Berlin, 1891.

12. N. K. Roberts, The surface tension of mercury and the adsorption of water vapor and some saturated hydrocarbons on mercury, J. Chem. Soc., (1964), 1907.

13. J. Serrin, A symmetry problem in potential theory, Arch. Rational Mech. Anal., 43 (1971), 304-318.

14. D. Siegel, Height estimates for capillary surfaces, Pacific J. Math., preceding in this issue.

15. H. Wente, The stability of the axially symmetric drop, Pacific J. Math., preceding in this issue. 
Received June 15, 1979. This investigation was supported in part by the National Science Foundation. Part of the work was done while the author was visiting at Universität Bonn.

Stanford University

StANFORd, CA 94305 



\section{PACIFIC JOURNAL OF MATHEMATICS}

\section{EDITORS}

DONALD BABBITT (Managing Editor)

University of California

Los Angeles, CA 90024

Hugo Rossi

University of Utah

Salt Lake City, UT 84112

C. C. MOORE and ANDREW OGG

University of California

Berkeley, CA 94720
J. DUGUNDJI

Department of Mathematics

University of Southern California

Los Angeles, CA 90007

R. FinN and J. Milgram

Stanford University

Stanford, CA 94305

\section{ASSOCIATE EDITORS}
E. F. BECKENBACH
B. H. NeumanN
F. WOLF
K. YoSHIDA

\section{SUPPORTING INSTITUTIONS}

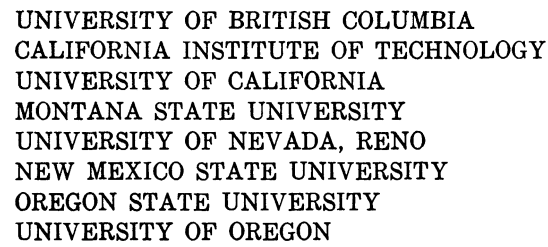

UNIVERSITY OF BRITISH COLUMBIA CALIFORNIA INSTITUTE OF TECHNOLOGY UNIVERSITY OF CALIFORNIA MONTANA STATE UNIVERSITY UNIVERSITY OF NEVADA, RENO NEW MEXICO STATE UNIVERSITY OREGON STATE UNIVERSITY UNIVERSITY OF OREGON

\author{
UNIVERSITY OF SOUTHERN CALIFORNIA \\ STANFORD UNIVERSITY \\ UNIVERSITY OF HAWAII \\ UNIVERSITY OF TOKYO \\ UNIVERSITY OF UTAH \\ WASHINGTON STATE UNIVERSITY \\ UNIVERSITY OF WASHINGTON
}

The Supporting Institutions listed above contribute to the cost of publication of this Journal, but they are not owners or publishers and have no responsibility for its content or policies.

Mathematical papers intended for publication in the Pacific Journal of Mathematics should be in typed form or offset-reproduced, (not dittoed), double spaced with large margins. Please do not use built up fractions in the text of the manuscript. However, you may use them in the displayed equations. Underline Greek letters in red, German in green, and script in blue. The first paragraph or two must be capable of being used separately as a synopsis of the entire paper. Please propose a heading for the odd numbered pages of less than 35 characters. Manuscripts, in triplicate, may be sent to any one of the editors. Please classify according to the scheme of Math. Reviews, Index to Vol. 39. Supply name and address of author to whom proofs should be sent. All other communications should be addressed to the managing editor, or Elaine Barth, University of California, Los Angeles, California, 90024.

50 reprints to each author are provided free for each article, only if page charges have been substantially paid. Additional copies may be obtained at cost in multiples of 50 .

The Pacific Journal of Mathematics is issued monthly as of January 1966. Regular subscription rate: $\$ 84.00$ a year (6 Vols., 12 issues). Special rato: $\$ 42.00$ a year to individual members of supporting institutions.

Subscriptions, orders for numbers issued in the last three calendar years, and changes of address shoud be sent to Pacific Journal of Mathematics, P.O. Box 969, Carmel Valley, CA 93924, U.S.A Old back numbers obtainable from Kraus Periodicals Co., Route 100, Millwood, NY 10546.

PUBLISHED BY PACIFIC JOURNAL OF MATHEMATICS, A NON-PROFIT CORPORATION

Printed at Kokusai Bunken Insatsusha (International Academic Printing Co., Ltd.). 8-8, 3-chome, Takadanobaba, Shinjuku-ku, Tokyo 160, Japan. 


\section{Pacific Journal of Mathematics \\ Vol. 88, No. $2 \quad$ April, 1980}

Reinhold Böhme, Stefan Hildebrandt and Engelbert Tausch, The two-dimensional analogue of the catenary ................. 247

Jean Ellen Taylor, Nonexistence of F-minimizing embedded disks ........ 279

Claus Gerhardt, A free boundary value problem for capillary surfaces ..... 285

Enrico Giusti, Generalized solutions for the mean curvature equation . . . . . 297

Jin-Tzu Chen, On the existence of capillary free surfaces in the absence of gravity.................................... 323

Leon M. Simon, Regularity of capillary surfaces over domains with corners.......................................... 363

Nicholas Jacob Korevaar, On the behavior of a capillary surface at a re-entrant corner.................................... 379

Henry Wente, The symmetry of sessile and pendent drops ............ 387

E. Gonzalez, Umberto Massari and I. Tamanini, Existence and regularity for the problem of a pendent liquid drop ...................... 399

Henry Wente, The stability of the axially symmetric pendent drop ........ 421

David Siegel, Height estimates for capillary surfaces . . . . . . . . . . . . 471

Bruce Edward Turkington, Height estimates for exterior problems of capillarity type ................................ 517

Robert Finn, The sessile liquid drop. I. Symmetric case .............. 541 